\title{
A New Unified Solution for Circular Opening considering Different Strength Criteria and the Postpeak Elastic Strain Form
}

\author{
Xuyang Shi $\mathbb{D}^{1},{ }^{1}$ Wei Zhou $\mathbb{D D}^{1}{ }^{1}$ Liang Chen $\mathbb{D}^{2},{ }^{2}$ Qingxiang Cai, ${ }^{1}$ Ming Li, ${ }^{2}$ Zhaolin Li, \\ and Boyu Luan ${ }^{1}$ \\ ${ }^{1}$ School of Mines, China University of Mining \& Technology, Xuzhou, Jiangsu 221116, China \\ ${ }^{2}$ State Key Laboratory for Geomechanics and Deep Underground Engineering, China University of Mining \& Technology, \\ Xuzhou, Jiangsu 221116, China \\ Correspondence should be addressed to Wei Zhou; loutian1982@126.com and Liang Chen; chenliang_cumt@126.com
}

Received 2 May 2020; Revised 17 July 2020; Accepted 25 August 2020; Published 30 September 2020

Academic Editor: Zhiqiang Yin

Copyright (c) 2020 Xuyang Shi et al. This is an open access article distributed under the Creative Commons Attribution License, which permits unrestricted use, distribution, and reproduction in any medium, provided the original work is properly cited.

\begin{abstract}
The strength criterion is an extremely important basis for evaluating the stability of surrounding rock and optimizing the support pressure design. In this paper, nine different strength criteria are summarized and simplified based on the reasonable assumption. Then, a new unified criterion equation is established, which includes all strength theories proposed by this paper. Meanwhile, a new unified closed-form solution for circular opening based on the newly proposed unified criterion equation is deduced with the infinite and finite external boundary combining with the nonassociative flow rule under plane strain conditions. In the plastic zone, four different elastic strain assumptions are applied to solving the plastic zone deformation considering the effect of rock mass damage. The solution's validity is also verified by comparison with the traditional solution. Finally, the influences of strength criteria, dilation coefficient, elastic strain form of plastic zone, and rock mass damage on the mechanical response of surrounding rock are discussed in detail. The research result shows that TR and VM criteria give the largest plastic zone radius, followed by IDP, $\mathrm{MC}$, and MDP criteria, and seem to underestimate the self-strength of rock mass; The CDP criterion gives the smallest plastic zone radius and may overestimate the self-strength of rock mass; UST0.5, GSMP, GMC, and GLD criteria that reasonably consider the effect of internal principal stresses give an intermediate range and can be strongly recommended for evaluating the mechanics and deformation behavior of surrounding rock; as the dilation coefficient gradually increases, the dimensionless surface displacement presents the nonlinear increase characteristics; the deformation of plastic zone and the ground response curve, which are closely related to the strength criteria, are also greatly influenced by the elastic strain assumption in the plastic zone and rock mass damage degree. The assumption that the elastic strain satisfies Hook's law (Case 3) may be more reasonable compared with the continuous elastic strain (Case 1) and thick-walled cylinders (Case 2) assumptions; in addition, the Young's modulus power function damage model seems to give more reasonable solution for the deformation of plastic zone and is suggested to be a preferred method for solving plastic displacement. The research results can provide very important theoretical bases for evaluating the tunnel stability and support design reliability of different lithology rock masses in underground engineering.
\end{abstract}

\section{Introduction}

Accurate prediction for the stress and displacement distribution of circular opening plays a crucial role in evaluating the mechanics and deformation behavior of rock mass in civil, mine, and oil engineering and natural gas development engineering. The circular opening may include tunnel, vertical shaft, boreholes, and pile foundation holes. In the early stage, many closed-form solutions of circular opening are obtained by regarding the outer boundary as the infinite boundary with association or nonassociative flow rules [1-9]. However, the semianalytical solutions considering the finite boundary are rarely deduced because of the complexity of the calculation process $[6,10-13]$. In practice, it is possible to calculate the outer boundary as an infinite boundary only if it is much larger than the diameter of circular opening. 
Therefore, this simplified calculation method has certain limitations in many geological engineering, such as shallowburied tunnel.

As for most of the analyses reported in the past, the solutions were given considering different yield criteria, such as linear Mohr-Coulomb (MC), Tresca (TR), and nonlinear Hoek-Brown (HB) criteria [2, 4-9, 14-17]. Nevertheless, the above studies did not consider the effect of intermediate principal stress on the mechanical response of surrounding rock. Many research results have shown that the intermediate principal stress exerts significant influence on the failure behavior of rock mass [6]. In addition, for rock mass as a natural geological material, the yield failure criterion is more complicated under the influence of internal crack and joint. Therefore, it is extremely difficult to reconcile the calculation results with the field measured results if only one or two yield criteria are used to predict the stresses and displacement behavior of surrounding rock. In this paper, as shown in Table 1, different yield criteria, such as Mogi-Coulomb (GMC) [9, 10], Drucker-Prager (CDP, MDP, and IDP) $[3,4,14,28-30]$, Generalized Lade-Duncan (GLD) [31, 35], Generalized SMP (GSMP) [18, 27, 31], and von Mises (VM) criteria $[15,26]$, will be summarized and simplified and then used to study the mechanical response of rock mass.

For an elastic-brittle plastic rock mass, the postpeak deformation is closely related to the assumed form of elastic strain in the plastic zone. Brown et al. researched the ground response curve for the rock tunnel by assuming that the elastic strain in the plastic region was equal to that on the elastic-plastic interface [36]. Sharan presented a series of new closed-form solutions for the prediction of displacements around circular openings in a brittle rock mass with nonlinear Hoek-Brown criterion by regarding the elastic strain of plastic zone as the thick-walled cylinder [16, 37]. Yu and Zhang and Reed et al. held that the elastic strain in the plastic zone satisfied the generalized Hooke's theorem and then derived the deformation of plastic zone with nonassociative flow laws $[9,38-40]$. In addition, Park summarized the above three different definitions for elastic strains in the plastic zone and analyzed the deformation law of plastic zone with Mohr-Coulomb and Hoek-Brown criteria under three different case conditions [9]. However, the postpeak Young's modulus attenuation along the radii direction is ignored in this study.

In this paper, different yield criteria of rock mass are firstly summarized and then a unified yield criterion form is derived by simplifying the above criteria. Next, a new closedform solution for stresses and deformation distribution around a circular opening subjected to the hydrostatic pressure at the finite or infinite outer boundary is also obtained with the new proposed unified yield criterion. In the plastic zone, five different definitions for elastic strains in the plastic zone and nonassociated flow rule are adopted to establish the radii displacement solution. The correctness of the solution is also verified by comparison with a series of traditional solution and numerical simulation results. Finally, the influences of strength theory and elastic strain definitions of plastic zone on the mechanical response of surrounding rock are discussed in detail.

\section{Brief Description of Yield Criterion}

2.1. Mohr-Coulomb Criterion. The linear Mohr-Coulomb criterion is widely used in geotechnical engineering because of its simple expression form. However, the influence of intermediate principal stress on rock mass failure is ignored. In addition, the triaxial strength of rock mass obtained by MC criterion deviates greatly from the measured data under the high confining pressure conditions. The following is the governing equation for $\mathrm{MC}$ criterion based on the cohesion $c$ and internal friction angle $\varphi[1,14,39]$ :

$$
\sigma_{1}=N \sigma_{3}+\sigma_{c}
$$

where $N$ is a constant which is a function of the internal friction angle and $\sigma_{c}$ is the uniaxial compressive strength (UCS). They can be expressed as follows:

$$
\begin{gathered}
N=\frac{(1+\sin \varphi)}{(1-\sin \varphi)}, \\
\sigma_{c}=\frac{(2 c \cos \varphi)}{(1-\sin \varphi)} .
\end{gathered}
$$

2.2. Tresca Criterion. The Tresca (TR) criterion is a simplified form of MC criterion and assumes that the failure will occur if the maximum shear stress $\tau_{\max }$ inside any plane of rock mass reaches a critical value $[1,15]$. As shown in Figure 1, the yield curve of TR criterion is a regular hexagon, which is inscribed in the circular yield curve of the von Mises (VM) criterion in $\pi$-plane. The expression form is

$$
\left(\frac{\sigma_{1}-\sigma_{3}}{2}\right)=\tau_{\max }=c
$$

The TR criterion can be considered as a special case where the internal friction angle equals zero in the MC criterion. Equation (2) can be rewritten as

$$
f\left(\sigma_{1}, \sigma_{3}\right)=\sigma_{1}-\sigma_{3}-2 c=0 .
$$

2.3. Generalized Lade-Duncan Criterion. In early 1973, the Lade-Duncan criterion has been firstly proposed by considering the intermediate principal stresses based on the triaxial compression test for noncohesive soil. The initial expression form can be written as follows:

$$
\frac{\left(I_{1}\right)^{3}}{I_{3}}=K_{\text {Lade }}
$$

where $I_{1}$ and $I_{3}$ are, respectively, the first and third principal stress invariants of friction material. $K_{\text {Lade }}$ is the material constant which is closely related to internal friction angle:

$$
\begin{aligned}
K_{\text {Lade }} & =\frac{(3-\sin \varphi)^{3}}{(1+\sin \varphi)(1-\sin \varphi)^{2}}, \\
I_{1} & =\sigma_{1}+\sigma_{2}+\sigma_{3},
\end{aligned}
$$




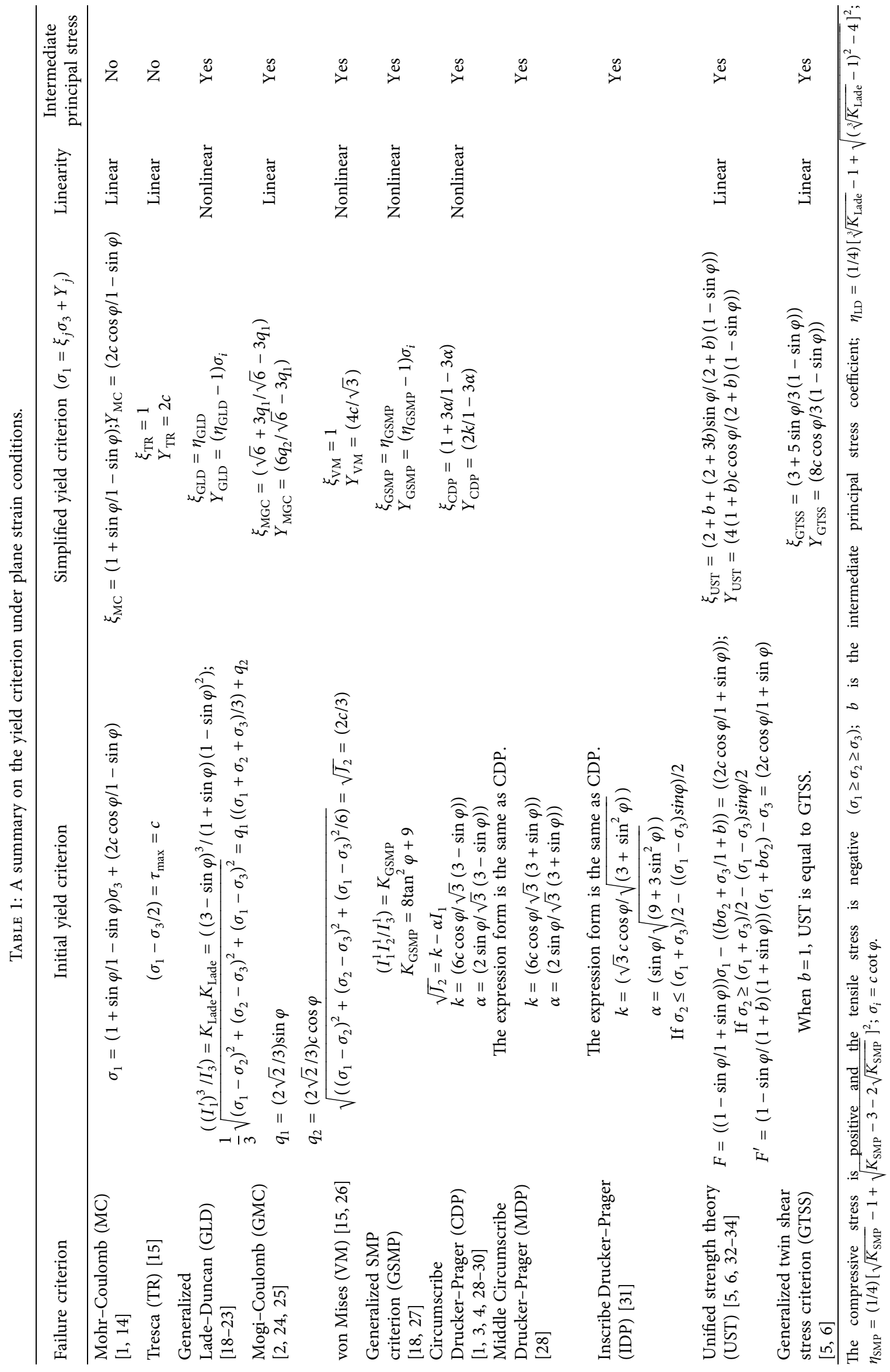




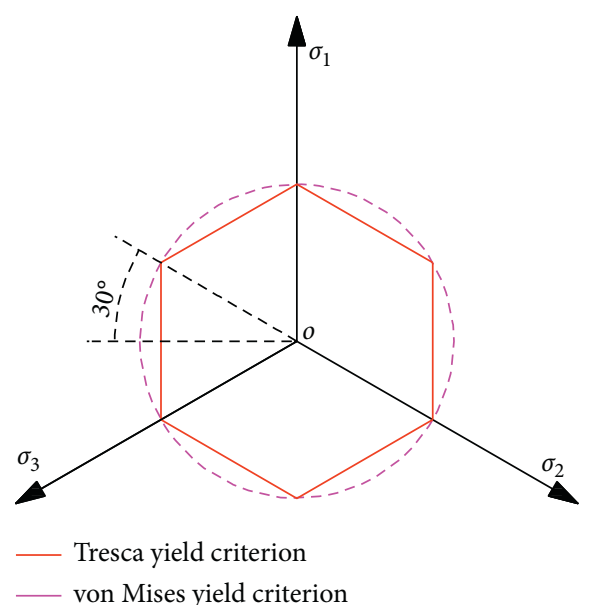

Figure 1: Tresca and von Mises criterion in the $\pi$-plane.

$$
I_{3}=\sigma_{1} \sigma_{2} \sigma_{3} .
$$

In 1999, the Lade-Duncan criterion was modified by Ewy so that it could reasonably describe the strength characteristics of cohesive soil by introducing bound stress $\left(\sigma_{0}\right)$ [35]. Its generalized expression is also given as

$$
\frac{\left(I_{1}^{\prime}\right)^{3}}{I_{3}^{\prime}}=K_{\text {Lade }}
$$

where $I_{1}^{\prime}$ and $I_{3}^{\prime}$ are, respectively, the first and third principal stress invariants of cohesive-friction material:

$$
\begin{aligned}
& I_{1}^{\prime}=\left(\sigma_{1}+\sigma_{0}\right)+\left(\sigma_{2}+\sigma_{0}\right)+\left(\sigma_{3}++\sigma_{0}\right), \\
& I_{3}^{\prime}=\left(\sigma_{1}+\sigma_{0}\right)\left(\sigma_{2}+\sigma_{0}\right)\left(\sigma_{3}++\sigma_{0}\right),
\end{aligned}
$$

where $\sigma_{0}$ is the bound stress: $\sigma_{0}=c \cot \varphi$. For the noncohesive soils, under plane strain conditions, the internal principal stress can be expressed as

$$
\sigma_{2}=\sqrt{\sigma_{1} \sigma_{3}} .
$$

By substituting equations (7), (8), and (11) into equation (6), the Lade-Duncan criterion can be rewritten as

$$
\sigma_{1}=\left(\frac{1}{4}\right)\left[\sqrt[3]{K_{\mathrm{Lade}}}-1+\sqrt{\left(\sqrt[3]{K_{\mathrm{Lade}}}-1\right)^{2}-4}\right]^{2} \sigma_{3}=\eta_{\mathrm{Lade}} \sigma_{3} .
$$

For the cohesive-friction material, according to the coordinate translation method [18, 19], the generalized Lade-Duncan (GLD) criterion will be obtained by integrating equation (12).

$$
\begin{aligned}
\sigma_{1}+\sigma_{0}= & \left(\frac{1}{4}\right)\left[\sqrt[3]{K_{\text {Lade }}}-1+\sqrt{\left(\sqrt[3]{K_{\text {Lade }}}-1\right)^{2}-4}\right]^{2} \\
& \cdot\left(\sigma_{3}+\sigma_{0}\right)=\eta_{\text {Lade }}\left(\sigma_{3}+\sigma_{0}\right) . \\
f\left(\sigma_{1}, \sigma_{3}\right)= & \sigma_{1}-\eta_{\text {Lade }} \sigma_{3}-\left(\eta_{\text {Lade }}-1\right) \sigma_{0}=0 .
\end{aligned}
$$

2.4. Generalized SMP Criterion. Considering the effect of internal principal stress, H. Matsuoka and T. Nakai proposed the SMP criterion, which could be expressed by three principal stress invariants [31]:

$$
\left(\frac{I_{1} I_{2}}{I_{3}}\right)=K_{\mathrm{SMP}}
$$

where $I_{2}$ is the second principal stress invariant and $K_{\mathrm{SMP}}$ is the material constant in the SMP criterion:

$$
\begin{aligned}
K_{\mathrm{SMP}} & =8 \tan ^{2} \varphi+9, \\
I_{2} & =\sigma_{1} \sigma_{3}+\sigma_{1} \sigma_{2}+\sigma_{2} \sigma_{3} .
\end{aligned}
$$

For the frictional material, by introducing equations (7), (8), (11), and (16) into equation (14), another form of SMP criterion will be deduced.

$$
\begin{gathered}
\sigma_{1}=\left(\frac{1}{4}\right)\left[\sqrt{K_{\mathrm{SMP}}}-1+\sqrt{K_{\mathrm{SMP}}-3-2 \sqrt{K_{\mathrm{SMP}}}}\right]^{2} \\
\cdot \sigma_{3}=\eta_{\mathrm{GSMP}} \sigma_{3} .
\end{gathered}
$$

As the GLD criterion, according to the coordinate translation method, the generalized SMP criterion will be given as [18]

$$
\begin{aligned}
\sigma_{1}+\sigma_{0}=\left(\frac{1}{4}\right)\left[\sqrt{K_{\mathrm{SMP}}}-1+\sqrt{K_{\mathrm{SMP}}-3-2 \sqrt{K_{\mathrm{SMP}}}}\right]^{2} \\
\cdot\left(\sigma_{3}+\sigma_{0}\right)=\eta_{\mathrm{GSMP}}\left(\sigma_{3}+\sigma_{0}\right) . \\
f\left(\sigma_{1}, \sigma_{3}\right)=\sigma_{1}-\eta_{\mathrm{GSMP}} \sigma_{3}-\left(\eta_{\mathrm{GSMP}}-1\right) \sigma_{0}=0 .
\end{aligned}
$$

As shown in Figure 2, compared with the yield curve of MC criterion in the $\pi$-plane, the GLD and GSMP criteria are nonlinear and the cross-sectional area of GLD criterion is the largest, followed by the GSMP criterion.

2.5. Mogi-Coulomb Criterion. Based on Mog's theory [24], Al-Ajmi and Zimmerman found that the polyaxial test data could be fitted by linear relationships in $\tau_{\text {oct }}-\sigma_{m, 2}$ space.

$$
\begin{aligned}
\tau_{\mathrm{oct}} & =a \sigma_{m, 2}+b \\
\tau_{\mathrm{oct}} & =\left(\frac{1}{3}\right) \sqrt{\left(\sigma_{1}-\sigma_{2}\right)^{2}+\left(\sigma_{1}-\sigma_{3}\right)^{2}+\left(\sigma_{2}-\sigma_{3}\right)^{2}}, \\
\sigma_{m, 2} & =\left(\frac{\sigma_{1}+\sigma_{2}+\sigma_{3}}{3}\right)
\end{aligned}
$$

where $\sigma_{m, 2}$ is the mean normal stress and $\tau_{\text {oct }}$ is octahedral shear stress. The parameters $a$ and $b$ can be related exactly to the Coulomb strength parameter: 


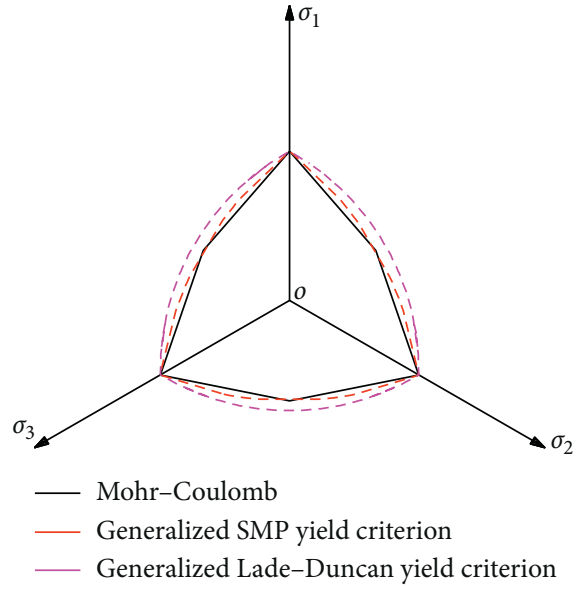

Figure 2: Comparison of MC, GLD, and GSMP criteria in the $\pi$-plane under plane strain condition.

$$
\begin{aligned}
& a=\left(\frac{2 \sqrt{2}}{3}\right) \sin \varphi, \\
& b=\left(\frac{2 \sqrt{2}}{3}\right) c \cos \varphi .
\end{aligned}
$$

As the von Mises criterion, we take $\sigma_{2}=\left(\left(\sigma_{1}+\sigma_{3}\right) / 2\right)$. Then, the Mogi-Coulomb (GMC) criterion can be obtained by integrating equations (19), (20), and (21).

$$
f\left(\sigma_{1}, \sigma_{3}\right)=\sigma_{1}-\left(\frac{\sqrt{6}+3 a}{\sqrt{6}-3 a}\right) \sigma_{3}-\left(\frac{6 b}{\sqrt{6}-3 a}\right)=0 .
$$

2.6. Unified Strength Theory. Based on the twin shear yield criterion, the unified strength theory (UST) is established by considering the influence of all the stress components on the material yield failure [32-34]. In geotechnical engineering, the cohesion and internal friction angle are usually used to represent this yield theory. The yield function can be expressed as follows $[5,6]$ :

$$
\begin{aligned}
& \text { If } \sigma_{2} \leq\left(\left(\sigma_{1}+\sigma_{3}\right) / 2\right)-\left(\left(\sigma_{1}-\sigma_{3}\right) / 2\right) \sin \varphi \\
& f\left(\sigma_{1}, \sigma_{3}\right)=\left(\frac{1-\sin \varphi}{1+\sin \varphi}\right) \sigma_{1}-\left(\frac{b \sigma_{2}+\sigma_{3}}{1+b}\right)=\left(\frac{2 c \cos \varphi}{1+\sin \varphi}\right)
\end{aligned}
$$

$$
\begin{aligned}
& \text { If } \sigma_{2} \leq\left(\left(\sigma_{1}+\sigma_{3}\right) / 2\right)-\left(\left(\sigma_{1}-\sigma_{3}\right) / 2\right) \sin \varphi \\
& f\left(\sigma_{1}, \sigma_{3}\right)=\frac{1-\sin \varphi}{(1+b)(1+\sin \varphi)}\left(\sigma_{1}+b \sigma_{2}\right)-\sigma_{3}=\left(\frac{2 c \cos \varphi}{1+\sin \varphi}\right) .
\end{aligned}
$$

where $b$ represents the yield parameter related to the intermediate principal stress, which can reflect the effect of the intermediate principal shear stress and the positive stress on the yield failure of the rock material, and $0 \leq b \leq 1$. As shown in Figure 3 , if $b=0$, UST will translate into the MC criterion; if $b=1$, UST is converted into general twin shear strength

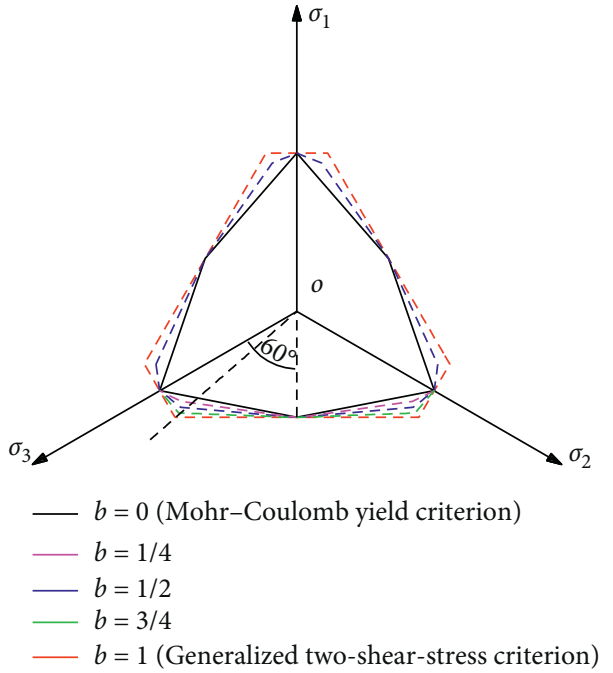

FIGURE 3: Unified strength theory yield curve in the $\pi$-plane [16].

(GTSS) criterion; if $0<b<1$, UST is a series of other ordered new strength criteria.

Just like Sections 2.3 and 2.6, if $\sigma_{2}=\left(\left(\sigma_{1}+\sigma_{3}\right) / 2\right)$, $\sigma_{2} \geq\left(\left(\sigma_{1}+\sigma_{3}\right) / 2\right)-\left(\left(\sigma_{1}-\sigma_{3}\right) / 2\right) \sin \varphi$ can be judged by substituting $\sigma_{2}=\left(\left(\sigma_{1}+\sigma_{3}\right) / 2\right)$ into equation $(24 \mathrm{a})$ or equation (24b). Therefore, UST can be rewritten as

$$
\begin{gathered}
f\left(\sigma_{1}, \sigma_{3}\right)=\sigma_{1}-\frac{2+b+(2+3 b) \sin \varphi}{(2+b)(1-\sin \varphi)} \sigma_{3} \\
-\frac{4(1+b) c \cos \varphi}{(2+b)(1-\sin \varphi)}=0 .
\end{gathered}
$$

2.7. Drucker-Prager Criterion. The Drucker-Prager (DP) criterion is an extension of the von Mises criterion, which takes into account the effect of intermediate principal stress and hydrostatic pressure on yield failure of the materials. It can be expressed as

$$
f\left(I_{1}, J_{2}\right)=\sqrt{J_{2}+\alpha I_{1}-k}=0,
$$

where the parameters $\alpha$ and $k$ are the material constants, which can be determined from the slope and the intercept of the failure envelope. The parameter $k$ is related to the cohesion and internal friction angle of rock mass. The parameter $\alpha$ is only related to the friction angle. Therefore, the Mohr-Coulomb parameter can be used to depict the DP criterion. By comparison with the yield curve of MC criterion in the $\pi$-plane (see Figure 4 ), the DP criteria can be divided into Circumscribe Drucker-Prager (CDP) criterion, Middle Circumscribe Drucker-Prager (MDP) criterion, and Inscribe Drucker-Prager (IDP) criterion.

(i) When the yield curve of DP criterion is the circumcircle of MC criterion, the material parameters $\alpha$ and $k$ for the CDP criterion can be obtained by $[4,28]$ 


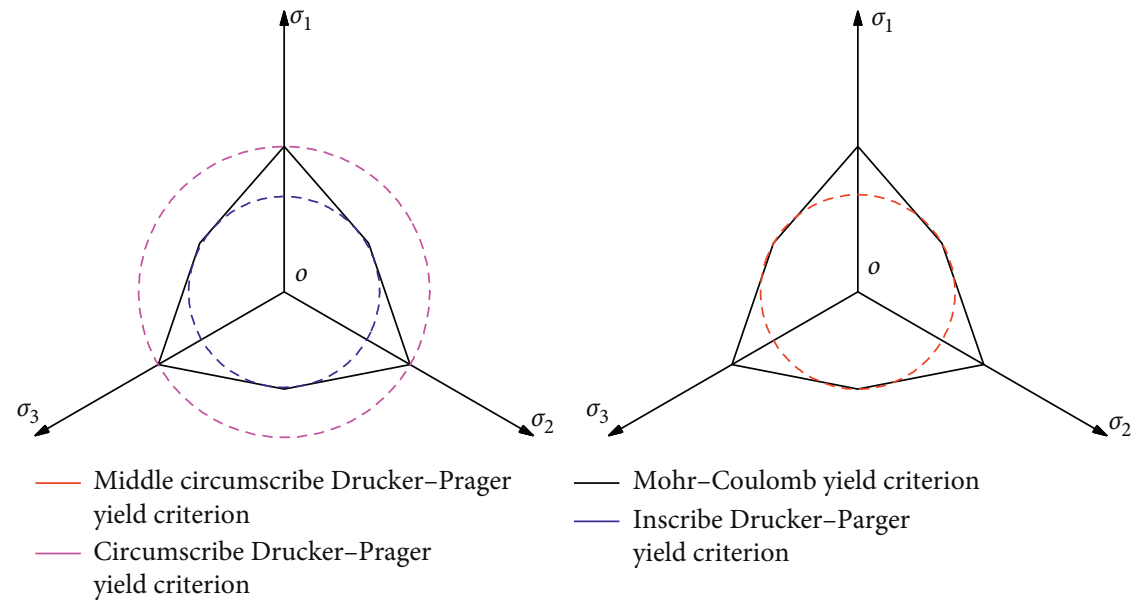

FIgURE 4: Drucker-Prager yield criterion curve in the $\pi$-plane.

$$
\begin{aligned}
& \alpha=\frac{2 \sin \varphi}{\sqrt{3}(3-\sin \varphi)}, \\
& k=\frac{6 c \cos \varphi}{\sqrt{3}(3-\sin \varphi)} .
\end{aligned}
$$

(ii) When the yield curve of DP criterion is the inscribed circle of MC criterion, the solution of parameters $\alpha$ and $k$ for the IDP criterion presented by Vekeens et al. [31] is

$$
\begin{aligned}
& \alpha=\frac{\sin \varphi}{\sqrt{\left(9+3 \sin ^{2} \varphi\right)}}, \\
& k=\frac{\sqrt{3} c \cos \varphi}{\sqrt{\left(3+\sin ^{2} \varphi\right)}} .
\end{aligned}
$$

(iii) When the yield curve of DP criterion is between CDP and IDP, the material parameters $\alpha$ and $k$ for the MDP criterion are expressed as follows:

$$
\begin{aligned}
& \alpha=\frac{2 \sin \varphi}{\sqrt{3}(3+\sin \varphi)}, \\
& k=\frac{6 c \cos \varphi}{\sqrt{3}(3+\sin \varphi)} .
\end{aligned}
$$

As shown in Sections 2.6 and 2.7, we take $\sigma_{2}=\left(\left(\sigma_{1}+\sigma_{3}\right) / 2\right)$. Then, the Drucker-Prager criterion can be deduced by solving equation (26).

$$
f\left(\sigma_{1}, \sigma_{3}\right)=\sigma_{1}-\left(\frac{1+3 \alpha}{1-3 \alpha}\right) \sigma_{3}-\left(\frac{2 k}{1-3 \alpha}\right)=0
$$

2.8. Establishment of Unified Criterion Equation. As shown in Table 1, the unified equation of different yield criterion can be summarized based on the above analysis under plane strain conditions.

$$
f\left(\sigma_{1}, \sigma_{3}\right)=\left(\sigma_{1}-\xi_{j}\right)\left(\sigma_{3}-Y_{j}\right)=0,
$$

where the subscript “ $j$ " represents different yield criteria and $\xi_{j}$ and $Y_{j}$ are the material constants of different criteria, which can be divided into two different cases. When $\xi_{j}=1$, equation (29) corresponds to the TR and VM criteria; when $\xi_{j} \neq 1$, equation (29) represents other criteria. Therefore, it can be regarded as a unified criterion equation to research the mechanical response of rock mass.

\section{Problem Description}

3.1. Establishment of Calculating Model. Figure 5 shows that a circular opening is excavated in a finite, isotropic, homogeneous elastic-brittle plastic rock mass subjected to an inner pressure $\sigma_{0}$ at the inner radii $R_{0}$ and a hydrostatic pressure $p_{0}$ at the external radius $R_{2}$. As $\sigma_{0}$ gradually decreases, the displacement will occur and the plastic zone with the radii $R_{1}$ will firstly develop around the circular opening when the maximum principal stress and minimum principal stress satisfy the initial yield condition. The influence of the rock mass weight in the plastic zone on the radial displacement and inner pressure is ignored. In this paper, the brittle plastic rock mass is introduced to research the postpeak mechanical behavior of rock material. As shown in Figure 6, the strength of the rock mass suddenly drops after peak load and then the postpeak softening behavior of strength parameters will occur. In other words, the postpeak cohesion $c_{\text {res }}$, internal friction angle $\varphi_{\text {res }}$, Young's modulus $E_{\text {res }}$, and Poisson's ratio $\nu_{\text {res }}$ are used to solve the stress and displacement distributions in the plastic zone.

Under the axisymmetric plane strain conditions, when $p_{0}>\sigma_{0}$, the hoop stress $\sigma_{\theta}$ and radial stress $\sigma_{r}$ are, respectively, maximum principal stress and minimum principal stress; the tangential strain $\varepsilon_{\theta}$ and radial strain $\varepsilon_{r}$ are, respectively, maximum principal strain and minimum principal strain. Accordingly, the unified criterion equation can be rewritten as follows: 


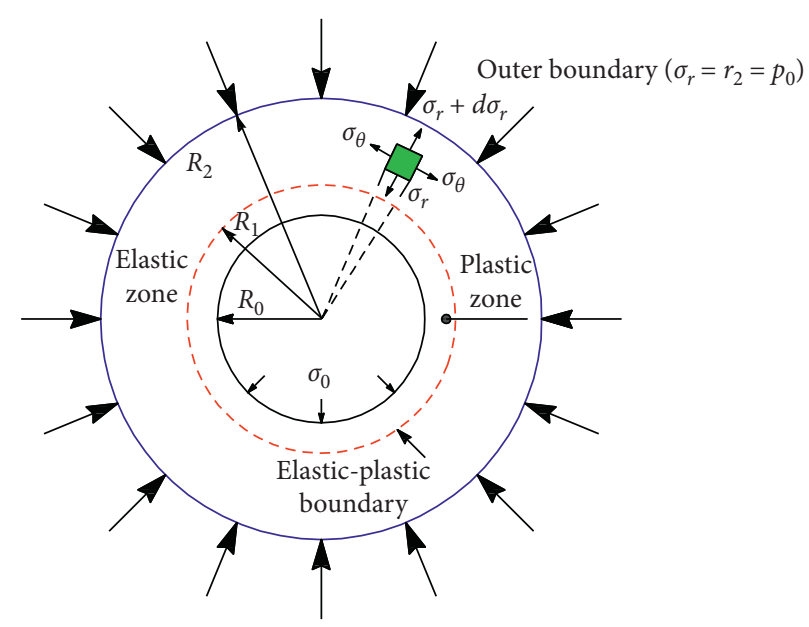

FIGURE 5: Calculation model of the circular opening with finite or infinite boundary.

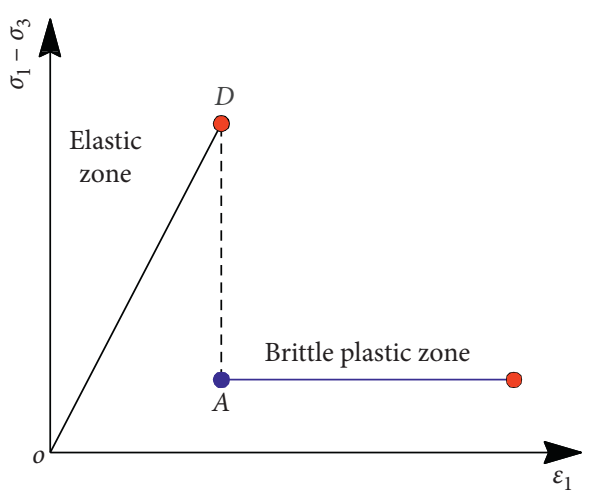

(a)

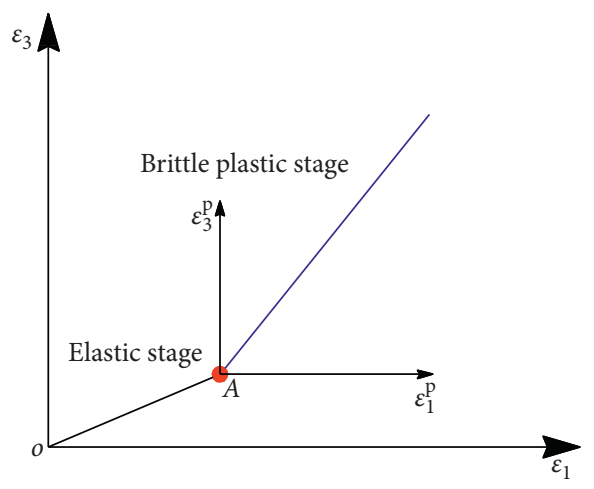

(b)

FIGURE 6: Postpeak failure behavior of brittle plastic rock mass.

$$
\begin{aligned}
& \sigma_{\theta(i)}=\xi_{j}^{\mathrm{ini}} \sigma_{r(i)}+Y_{j}^{\mathrm{ini}} \text { for peak stage, } \\
& \sigma_{\theta(i)}=\xi_{j}^{\mathrm{res}} \sigma_{r(i)}+Y_{j}^{\mathrm{res}} \text { for postpeak stage, }
\end{aligned}
$$

where the subscript “” represents different zones $(i=1,2)$, $\xi_{j}^{\text {ini }}$ and $Y_{j}^{\text {ini }}$ are the initial strength parameters, respectively, and $\xi_{j}^{\text {res }}$ and $Y_{j}^{\text {res }}$ are the residual strength parameters, respectively.

3.2. Based Equation. For the axisymmetric plane strain problem, the equilibrium differential equation can be expressed as (ignoring the body force of rock masses) $[29,30,39]$

$$
\left(\frac{d \sigma_{r(i)}}{d r}\right)+\left(\frac{\sigma_{r(i)}-\sigma_{\theta(i)}}{r}\right)=0 .
$$

The geometric equation, based on the small deformation assumption, can be denoted as

$$
\begin{aligned}
& \varepsilon_{r(i)}=\left(\frac{\partial u_{r(i)}}{\partial r}\right), \\
& \varepsilon_{\theta(i)}=\left(\frac{u_{r(i)}}{r}\right),
\end{aligned}
$$

where $u_{r(i)}$ is the radial displacement. Both the radial displacement $u_{R_{1}}$ and radial contact stress $\sigma_{R_{1}}$ should be continuous at the elastic-plastic interface, respectively. Therefore, the boundary conditions around the circular opening can be summarized as

$$
\left\{\begin{array}{l}
r=R_{0}, \sigma_{r(1)}=\sigma_{0}, \\
r=R_{1}, \sigma_{r(1)}=\sigma_{R_{1}}, u_{r(1)}=u_{R_{1}}, \\
r=R_{2}, \sigma_{r(2)}=p_{0} .
\end{array}\right.
$$




\section{The New Unified Solution of Circular Opening}

4.1. A Semianalytical Solution with the Finite External Boundary. According to the elastic mechanics theory, the stresses and displacement in the elastic zone can be deduced by regarding it as a hollow thick-wall cylinder subjected to an inner pressure $\sigma_{R_{1}}$ at the internal boundary $r=R_{1}$ and a hydrostatic pressure $p_{0}$ at the external boundary $r=R_{2}$ as follows:

$$
\begin{aligned}
\sigma_{r(2)}= & \frac{\left(R_{2} R_{1}\right)^{2}\left(\sigma_{R_{1}}-p_{0}\right)}{R_{2}^{2}-R_{1}^{2}}\left(\frac{1}{r^{2}}\right)+\left(\frac{R_{2}^{2} p_{0}-R_{1}^{2} \sigma_{R_{1}}}{R_{2}^{2}-R_{1}^{2}}\right), \\
\sigma_{\theta(2)}= & -\frac{\left(R_{2} R_{1}\right)^{2}\left(\sigma_{R_{1}}-p_{0}\right)}{R_{2}^{2}-R_{1}^{2}}\left(\frac{1}{r^{2}}\right)+\left(\frac{R_{2}^{2} p_{0}-R_{1}^{2} \sigma_{R_{1}}}{R_{2}^{2}-R_{1}^{2}}\right), \\
u_{r(2)=} & \left(\frac{1-v-2 v^{2}}{E}\right)\left(\frac{R_{2}^{2} p_{0}-R_{1}^{2} \sigma_{R_{1}}}{R_{2}^{2}-R_{1}^{2}}\right) r \\
& +\left(\frac{1+v}{E}\right) \frac{\left(R_{2} R_{1}\right)^{2}\left(p_{0}-\sigma_{R_{1}}\right)}{R_{2}^{2}-R_{1}^{2}}\left(\frac{1}{r}\right)
\end{aligned}
$$

where $E$ and $v$ are the initial Young's modulus and initial Poisson's ratio, respectively. The initial yield failure condition should be satisfied at the elastic-plastic interface. Therefore, the radial contact stress $\sigma_{R_{1}}$ can be derived by substituting equations (34a) and (34b) into equation (30a) under different yield criteria:

$$
\sigma_{R_{1}}=\frac{\left(2 p_{0}-Y_{j}^{\mathrm{ini}}\right) R_{2}^{2}+R_{1}^{2} Y_{j}^{\mathrm{ini}}}{\left(1+\xi_{j}^{\mathrm{ini}}\right) R_{2}^{2}+\left(1-\xi_{j}^{\mathrm{ini}}\right) R_{1}^{2}} .
$$

Obviously, the stresses in the plastic zone should satisfy the equilibrium differential equation and are easily deduced by submitting equation (30b) into equation (31) as well as combining with the boundary condition $\sigma_{r(1)}=\sigma_{0}$ at $r=R_{0}$. However, for $\xi_{j}^{\text {res }}=1$ and $\xi_{j}^{\text {res }} \neq 1$, the stress solutions in the plastic zone are inconsistent.

(i) $\mathrm{f} \xi_{j}^{\text {res }} \neq 1$, the unified stresses solution in the plastic zone will be obtained as

$$
\begin{aligned}
\sigma_{r(1)} & =\left(\sigma_{0}+\frac{Y_{j}^{\mathrm{res}}}{\xi_{j}^{r e s}-1}\right)\left(\frac{r}{R_{0}}\right)^{\xi_{j}^{\mathrm{res}}-1}-\left(\frac{Y_{j}^{\mathrm{res}}}{\xi_{j}^{\mathrm{res}}-1}\right), \\
\sigma_{\theta(1)} & =\xi_{j}^{\mathrm{res}}\left(\sigma_{0}+\frac{Y_{j}^{\mathrm{res}}}{\xi_{j}^{\mathrm{res}}-1}\right)\left(\frac{r}{R_{0}}\right)^{\xi_{j}^{\mathrm{res}}-1}-\left(\frac{Y_{j}^{\mathrm{res}}}{\xi_{j}^{\mathrm{res}}-1}\right) .
\end{aligned}
$$

Here, the subscript “ $j$ ” represents MC, GMC, GLD, GSMP, CDP, MDP, IDP, UST, and GTSS criteria.
Then, the radii of plastic zone can be determined by considering the boundary condition $\sigma_{r(1)}=\sigma_{R_{1}}$ at the elastic-plastic interface. By integrating equation (35) and equation (36a), the nonlinear function between $R_{1}$ and $R_{2}$ can be established as follows:

$$
\begin{aligned}
& {\left[\left(1+\xi_{j}^{\mathrm{ini}}\right) R_{2}^{2}+\left(1-\xi_{j}^{\mathrm{ini}}\right) R_{1}^{2}\right]\left[\left(\sigma_{0}+\frac{Y_{j}^{\mathrm{res}}}{\xi_{j}^{\mathrm{res}}-1}\right)\right.} \\
& \left.\cdot\left(\frac{R_{1}}{R_{0}}\right)^{\xi_{j}^{\mathrm{res}}-1}-\frac{Y_{j}^{\mathrm{res}}}{\xi_{j}^{\mathrm{res}}-1}\right]=\left(2 p_{0}-Y_{j}^{\mathrm{ini}}\right) R_{2}^{2}+R_{1}^{2} Y_{j}^{\mathrm{ini}} .
\end{aligned}
$$

(ii) If $\xi_{j}^{\text {res }}=1$, the unified stress solutions of plastic zone based on the TR and VM criteria are

$$
\begin{aligned}
\sigma_{r(1)} & =\sigma_{0}+Y_{j}^{\mathrm{res}} \ln \left(\frac{r}{R_{0}}\right) \\
\sigma_{\theta(1)} & =\sigma_{0}+Y_{j}^{\mathrm{res}}\left[1+\ln \left(\frac{r}{R_{0}}\right)\right] .
\end{aligned}
$$

As the solution for equation (41), the nonlinear function between $R_{1}$ and $R_{2}$ based on the VM and TR criterion can be also deduced as follows:

$$
\begin{aligned}
& {\left[\left(1+\xi_{j}^{\mathrm{ini}}\right) R_{2}^{2}+\left(1-\xi_{j}^{\mathrm{ini}}\right) R_{1}^{2}\right]\left[\sigma_{0}+Y_{j}^{\mathrm{res}} \ln \left(\frac{R_{1}}{R_{0}}\right)\right]} \\
& \quad=\left(2 p_{0}-Y_{j}^{\mathrm{ini}}\right) R_{2}^{2}+R_{1}^{2} Y_{j}^{\mathrm{ini}} .
\end{aligned}
$$

In practice, the external radius $R_{2}$ is a constant. The approximate solution for $R_{1}$ in equations (37) and (39) can be obtained by using the numerical calculation methods such as the least square method and Newton iteration method. Then, the stresses and displacement in elastic zone can be determined by equations (34a), (34b), and (34c).

In the plastic zone, the total hoop strain $\varepsilon_{\theta(1)}$ and radial strain $\varepsilon_{r(1)}$ are, respectively, composed of elastic strain and plastic strain. Therefore, the total strain can be expressed as

$$
\left\{\begin{array}{l}
\varepsilon_{\theta(1)}=\varepsilon_{\theta(1)}^{e}+\varepsilon_{\theta(1)}^{p}, \\
\varepsilon_{r(1)}=\varepsilon_{r(1)}^{e}+\varepsilon_{r(1)}^{p},
\end{array}\right.
$$

where $\varepsilon_{\theta(1)}^{e}$ and $\varepsilon_{r(1)}^{e}$ are, respectively, the hoop elastic strain and radial elastic strain in the plastic zone; $\varepsilon_{\theta(1)}^{p}$ and $\varepsilon_{r(1)}^{p}$ are the hoop plastic strain and radial plastic strain of plastic zone, respectively.

For axisymmetric plane strain problems, the plastic strain relationships can be established by considering the small strain theory and nonassociated flow rule $[5,9]$.

$$
\varepsilon_{r(1)}^{p}+\beta \varepsilon_{\theta(1)}^{p}=0
$$


where $\quad \beta$ is the dilation coefficient: $\beta=(1+\sin \psi) /(1-\sin \psi) . \psi$ is the dilation angle.

By substituting equations (32) and (41) into equation (44), the following differential equation for the radial displacement in the plastic zone can be derived as

$$
\left(\frac{\partial u_{r(1)}}{\partial r}\right)+\beta\left(\frac{u_{r(1)}}{r}\right)=\varepsilon_{r(1)}^{e}+\beta \varepsilon_{\theta(1)}^{e}=f(r) .
$$

From equation (42), it can be seen that the radial displacement is closely related to the elastic strain form in the plastic zone.

Then, the following function can be obtained by solving equation (42) as follows:

$$
u_{r(1)}=\frac{1}{r^{\beta}} \int_{R_{1}}^{r} f(r) r^{\beta} d r+u_{R_{1}}\left(\frac{R_{1}}{r}\right)^{\beta},
$$

where $u_{R_{1}}$ is the radial displacement at the elastic-plastic interface. It can be easily determined by taking $r=R_{1}$ in equation (34c) as follows:

$$
\begin{aligned}
u_{R_{1}}= & \left(\frac{1-v-2 v^{2}}{E}\right)\left(\frac{R_{2}^{2} p_{0}-R_{1}^{2} \sigma_{R_{1}}}{R_{2}^{2}-R_{1}^{2}}\right) R_{1} \\
& +\left(\frac{1+v}{E}\right)\left(\frac{R_{2}^{2} R_{1}\left(p_{0}-\sigma_{R_{1}}\right)}{R_{2}^{2}-R_{1}^{2}}\right) .
\end{aligned}
$$

In order to obtain the radial displacement of plastic zone, the expression for elastic strain should be firstly determined. Generally, four different definitions for elastic strain can be used to research the deformation behavior of rock mass in the plastic zone.

(1) Case 1: it is assumed that the elastic strain in the plastic region is equal to that on the elastic-plastic interface. Then, the elastic strains can be expressed as [36]

$$
\left\{\begin{array}{l}
\varepsilon_{\theta(1)}^{e}=\left(\frac{u_{r(2)}}{r}\right)_{r=R_{1}}=\left(\frac{1-v-2 v^{2}}{E}\right)\left(\frac{R_{2}^{2} p_{0}-R_{1}^{2} \sigma_{R_{1}}}{R_{2}^{2}-R_{1}^{2}}\right)+\left(\frac{1+\nu}{E}\right)\left(\frac{R_{2}^{2}\left(p_{0}-\sigma_{R_{1}}\right)}{R_{2}^{2}-R_{1}^{2}}\right) . \\
\varepsilon_{r(1)}^{e}=\left(\frac{\partial u_{r(2)}}{\partial r}\right)_{r=R_{1}}=\left(\frac{1-v-2 v^{2}}{E}\right)\left(\frac{R_{2}^{2} p_{0}-R_{1}^{2} \sigma_{R_{1}}}{R_{2}^{2}-R_{1}^{2}}\right)-\left(\frac{1+\nu}{E}\right)\left(\frac{R_{2}^{2}\left(p_{0}-\sigma_{R_{1}}\right)}{R_{2}^{2}-R_{1}^{2}}\right) .
\end{array}\right.
$$

Then, the function $f(r)$ is

$$
\begin{aligned}
f(r)= & \left(\frac{\left(1-\nu-2 \nu^{2}\right)(1+\beta)}{E}\right)\left(\frac{R_{2}^{2} p_{0}-R_{1}^{2} \sigma_{R_{1}}}{R_{2}^{2}-R_{1}^{2}}\right) \\
& -\left(\frac{(1+\nu)(1-\beta)}{E}\right)\left(\frac{R_{2}^{2}\left(p_{0}-\sigma_{R_{1}}\right)}{R_{2}^{2}-R_{1}^{2}}\right)=\delta_{\text {case } 1} .
\end{aligned}
$$

By substituting equation (46) into equation (43), the radial displacement in the plastic zone can be derived as follows:

$$
u_{r(1)}^{\text {case1 }}=\left(\frac{\delta_{\text {casel }}}{(1+\beta)}\right)\left(\frac{r^{\beta+1}-R_{1}^{\beta+1}}{r^{\beta}}\right)+u_{R_{1}}\left(\frac{R_{1}}{r}\right)^{\beta} .
$$

(2) Case 2: by regarding the plastic zone as the thick-wall cylinder subjected to the inner pressure $\sigma_{0}$ at $r=R_{0}$ and radial contact stress $\sigma_{R_{1}}$ at $r=R_{0}$, the elastic strain in the plastic zone can be written as $[19,39]$

$$
\left\{\begin{array}{l}
\varepsilon_{\theta(1)}^{e}=\frac{1+v_{\text {res }}}{E_{\text {res }}}\left[\left(1-2 v_{\text {res }}\right) C_{1}-\left(\frac{C_{2}}{r^{2}}\right)\right], \\
\varepsilon_{r(1)}^{e}=\left(\frac{1+v_{\text {res }}}{E_{\text {res }}}\right)\left[\left(1-2 v_{\text {res }}\right) C_{1}+\frac{C_{2}}{r^{2}}\right],
\end{array}\right.
$$

where $C_{1}=\left(\left(\sigma_{R_{1}}-p_{0}\right) R_{1}^{2}-\left(\sigma_{0}-p_{0}\right) R_{0}^{2} / R_{1}^{2}-R_{0}^{2}\right)$ and $C_{2}=\left(R_{1}^{2} R_{0}^{2}\left(\sigma_{0}-\sigma_{R_{1}}\right) / R_{1}^{2}-R_{0}^{2}\right)$. Therefore, the function $f(r)$ is

$$
\begin{aligned}
f(r) & =\left(\frac{1+v_{\text {res }}}{E_{\text {res }}}\right)\left[(1+\beta)\left(1-2 v_{\text {res }}\right) C_{1}+(1-\beta) \frac{C_{2}}{r^{2}}\right], \\
& =\delta_{1}^{\text {Case2 }}(1+\beta)+\delta_{2}^{\text {Case2 }}(1-\beta) r^{-2},
\end{aligned}
$$

where $\quad \delta_{1}^{\text {Case2 }}=\left(1+v_{\text {res }} / E_{\text {res }}\right)\left(1-2 v_{\text {res }}\right) C_{1} \quad$ and $\delta_{2}^{\text {Case2 }}=\left(1+v_{\text {res }} / E_{\text {res }}\right) C_{2}$.

By submitting equation (49) into equation (43), we can obtain the radial displacement of plastic zone as follows: 


$$
\begin{aligned}
u_{r(1)}^{\text {case2 }}= & \left(\frac{1}{r^{\beta}}\right)\left[\delta_{1}^{\text {Case2 }}\left(r^{\beta+1}-R_{1}^{\beta+1}\right)-\delta_{2}^{\text {Case2 }}\left(r^{\beta-1}-R_{1}^{\beta-1}\right)\right] \\
& +u_{R_{1}}\left(\frac{R_{1}}{r}\right)^{\beta} .
\end{aligned}
$$

(3) Case 3: adopting Hooke's law, the elastic strains in the plastic zone can be written as follows [27]:

$$
\left\{\begin{array}{l}
\varepsilon_{\theta(1)}^{e}=\frac{\left(1+v_{\text {res }}\right)}{E_{\text {res }}}\left[\left(1-v_{\text {res }}\right) \sigma_{\theta(1)}-v_{\text {res }} \sigma_{r(1)}\right] . \\
\varepsilon_{r(1)}^{e}=\frac{\left(1+v_{\text {res }}\right)}{E_{\text {res }}}\left[\left(1-v_{r e s}\right) \sigma_{r(1)}-v_{\text {res }} \sigma_{\theta(1)}\right] .
\end{array}\right.
$$

Then, the function $f(r)$ can be expressed as follows:

$$
\begin{gathered}
f(r)=\frac{1+v_{\text {res }}}{E_{\text {res }}}\left[\left(1-v_{\text {res }}-\beta v_{\text {res }}\right) \sigma_{r(1)}\right. \\
\left.+\left(\beta-v_{\text {res }}-\beta v_{\text {res }}\right) \sigma_{\theta(1)}\right] .
\end{gathered}
$$

(i) If $\xi_{j}^{\text {res }} \neq 1$, the function $f(r)$ will be rewritten by substituting equations (36a) and (36b) into equation (52) as follows:

$$
f(r)=\left(\frac{1+v_{\text {res }}}{E_{\text {res }}}\right)\left[\delta_{1}^{\text {case3 }}\left(\frac{r}{R_{0}}\right)^{\xi_{j}^{\text {res }}-1}-\delta_{2}^{\text {case } 3}\right],
$$

where $\quad \delta_{1}^{\text {case3 }}=\left(\sigma_{0}+\left(Y_{j}^{\text {res }} / \xi_{j}^{\text {res }}-1\right)\right)\left[1+\beta \xi_{j}^{\text {res }}-\right.$ $\left.\left(1+\xi_{j}^{\text {res }}\right)\left(\nu_{\text {res }}+\beta \nu_{\text {res }}\right)\right]$ and $\delta_{2}^{\text {case } 3}=\left(1-2 v_{\text {res }}\right)$ $(1+\beta)\left(Y_{j}^{\mathrm{res}} / \xi_{j}^{\mathrm{res}}-1\right)$.

By substituting equation (53) into equation (43), the radial displacement in the plastic zone can be derived as follows:

$$
\begin{aligned}
u_{r(1)}^{\mathrm{case} 3}= & \left(\frac{1+v_{\mathrm{res}}}{E_{\mathrm{res}}}\right)\left(\frac{1}{r^{\beta}}\right)\left[\frac{\delta_{1}^{\mathrm{case} 3}}{\left(\xi_{j}^{\mathrm{res}}+\beta\right) R_{0}^{\xi_{j}^{\mathrm{res}}}-1}\left(r^{\xi_{j}^{\mathrm{res}}+\beta}-R_{1}^{\xi_{j}^{\mathrm{res}}+\beta}\right)\right. \\
& \left.-\frac{\delta_{2}^{\mathrm{case} 3}}{(1+\beta)}\left(r^{1+\beta}-R_{1}^{1+\beta}\right)\right]+u_{R_{1}}\left(\frac{R_{1}}{r}\right)^{\beta} .
\end{aligned}
$$

(ii) If $\xi_{j}^{\text {res }}=1$, the function $f(r)$ can be obtained by substituting equations (38a) and (38b) into equation (52) as follows:

$$
f(r)=\left(\frac{1+v_{\text {res }}}{E_{\text {res }}}\right)\left[\delta_{3}^{\text {case3 }} \ln \left(\frac{r}{R_{0}}\right)+\delta_{4}^{\text {case } 3}\right]
$$

where $\delta_{3}^{\text {case } 3}=\left(1-2 v_{\text {res }}\right)(1+\beta) Y_{j}^{\text {res }}$ and $\delta_{4}^{\text {case3 }}=(1-$ $\left.v_{\text {res }}-\beta v_{\text {res }}\right) \sigma_{0}+\left(\beta-v_{\text {res }}-\beta v_{\text {res }}\right)\left(\sigma_{0}+Y_{j}^{\text {res }}\right)$.
By submitting equation (55) to equation (43), the radial displacements for TR and VM criterion in the plastic zone are

$$
\begin{aligned}
u_{r(1)}^{\text {case3 }}= & \left(\frac{1+v_{\text {res }}}{E_{\text {res }}}\right)\left(\frac{1}{r^{\beta}}\right)\left\{\frac{\delta_{3}^{\text {case3 }}}{1+\beta}\left[r^{1+\beta} \ln \left(\frac{r}{R_{0}}\right)-R_{1}^{1+\beta} \ln \left(\frac{R_{1}}{R_{0}}\right)\right]\right. \\
& \left.+\left[\frac{\delta_{4}^{\text {case3 }}}{1+\beta}-\frac{\delta_{3}^{\text {case3 }}}{(1+\beta)^{2}}\right]\left(r^{1+\beta}-R_{1}^{1+\beta}\right)\right\}+u_{R_{1}}\left(\frac{R_{1}}{r}\right)^{\beta} .
\end{aligned}
$$

(4) Case 4: the mechanical behavior of rock mass is closely related to its damage degree in the plastic zone. The higher damage usually leads to larger deformation behavior. Then, the attenuation of Young's modulus should be considered in the plastic zone. In this case, the power function attenuation model of Young's modulus along the radius direction is introduced to research the deformation behavior in the plastic zone [6]:

$$
\begin{gathered}
E(r)=E_{\mathrm{res}}\left(\frac{r}{R_{0}}\right)^{m} . \\
m=\frac{\log \left(E / E_{\mathrm{res}}\right)}{\log \left(R_{1} / R_{0}\right)} .
\end{gathered}
$$

(i) If $\xi_{j}^{\text {res }} \neq 1$, the function $f(r)$ will be rewritten by substituting equation (57) into equation (53) as follows:

$$
f(r)=\left(\frac{1+v_{\mathrm{res}}}{E_{\mathrm{res}}}\right)\left(\frac{R_{0}}{r}\right)^{m}\left[\delta_{1}^{\text {case } 3}\left(\frac{r}{R_{0}}\right)^{\xi_{j}^{\text {res }}-1}-\delta_{2}^{\text {case } 3}\right] \text {. }
$$

By substituting equation (58) into equation (43), the radial displacement in the plastic zone can be derived. In fact, the expression form is the same as that in equation (54):

$$
\begin{aligned}
u_{r(1)}^{\text {case3 }}= & \left(\frac{1+v_{\text {res }}}{E_{\text {res }}}\right) \frac{1}{r^{\beta}}\left[\left(\frac{\delta_{1}^{\text {case } 3}}{\left(\xi_{j}^{\text {res }}+\beta-m\right) R_{0}^{\xi_{j}^{\text {res }}-m-1}}\right)\right. \\
& \cdot\left(r^{\xi_{j}^{\text {res }}+\beta-m}-R_{1}^{\xi_{j}^{\text {res }}+\beta-m}\right)-\left(\frac{\delta_{2}^{\text {case } 3}}{(1+\beta-m) R_{0}^{-m}}\right) \\
& \left.\cdot\left(r^{1+\beta-m}-R_{1}^{1+\beta-m}\right)\right]+u_{R_{1}}\left(\frac{R_{1}}{r}\right)^{\beta} .
\end{aligned}
$$

(ii) If $\xi_{j}^{\text {res }}=1$, the function $f(r)$ can be obtained by substituting equation (57) into equation (55) as follows:

$$
f(r)=\left(\frac{1+v_{\text {res }}}{E_{\text {res }}}\right)\left(\frac{R_{0}}{r}\right)^{m}\left[\delta_{3}^{\text {case } 3} \ln \left(\frac{r}{R_{0}}\right)+\delta_{4}^{\text {case3 }}\right] .
$$


By introducing equation (60) to equation (43), the expression form for radial displacement is similar to equation (56):

$$
\begin{aligned}
u_{r(1)}^{\text {case3 }}= & \left(\frac{1+v_{\text {res }}}{E_{\text {res }}}\right) \frac{1}{r^{\beta}}\left\{\frac { \delta _ { 3 } ^ { \text { case3 } } R _ { 0 } ^ { m } } { 1 + \beta - m } \left[r^{1+\beta-m} \ln \left(\frac{r}{R_{0}}\right)\right.\right. \\
& \left.-R_{1}^{1+\beta-m} \ln \left(\frac{R_{1}}{R_{0}}\right)\right]+\left[\frac{\delta_{4}^{\text {case3 }}}{1+\beta-m}-\frac{\delta_{3}^{\text {case3 }}}{(1+\beta-m)^{2}}\right] \\
& \left.\cdot R_{0}^{m}\left(r^{1+\beta-m}-R_{1}^{1+\beta-m}\right)\right\}+u_{R_{1}}\left(\frac{R_{1}}{r}\right)^{\beta} .
\end{aligned}
$$

4.2. The Closed-Form Solution with the Infinite External Boundary. For the deep underground engineering, a circular opening subjected to an inner support pressure $\sigma_{0}$ at $r=R_{0}$ and hydrostatic pressure $p_{0}$ at infinite external boundary is a special case of the axisymmetric thick-wall cylinder. The stress and displacement solutions can be obtained through the same solving process as that in Section 4.1. When $R_{2} \longrightarrow \infty$, the stresses in the elastic zone can be easily deduced by equations (34a) and (34b) as follows:

$$
\begin{aligned}
& \sigma_{r(2)}=p_{0}-\left(p_{0}-\sigma_{R_{1}}\right)\left(\frac{R_{1}}{r}\right)^{2}, \\
& \sigma_{\theta(2)}=p_{0}+\left(p_{0}-\sigma_{R_{1}}\right)\left(\frac{R_{1}}{r}\right)^{2} .
\end{aligned}
$$

Accordingly, the stress distribution for deep circular opening in the plastic zone can be also calculated by equations (36a) and (36b) for $\xi_{j}^{\text {res }} \neq 1$ or equations (38a) and (38b) for $\xi_{j}^{\text {res }}=1$. In addition, the unified radial contact stress $\sigma_{R_{1}}$ for different criteria can be rewritten by taking $R_{2} \longrightarrow \infty$ in equation (35) as follows:

$$
\sigma_{R_{1}}=\frac{\left(2 p_{0}-Y_{j}^{\mathrm{ini}}\right)}{\left(1+\xi_{j}^{\mathrm{ini}}\right)} .
$$

As shown in equation (37), if $\xi_{j}^{\text {res }} \neq 1$, the radius of plastic zone for deep circular opening can be easily deduced as follows:

$$
R_{1}=R_{0} \sqrt[\xi_{j}^{\text {res }}]{\frac{\left(\left(2 p_{0}-Y_{j}^{\mathrm{ini}}\right) /\left(1+\xi_{j}^{\mathrm{ini}}\right)\right)+\left(Y_{j}^{\mathrm{res}} /\left(\xi_{j}^{\mathrm{res}}-1\right)\right)}{\left(\sigma_{0}+Y_{j}^{\mathrm{res}} /\left(\xi_{j}^{\mathrm{res}}-1\right)\right)}} .
$$

As shown in equation (39), if $\xi_{j}^{\text {res }}=1$, the unified radius solution of plastic zone based on TR and VM criteria is

$$
R_{1}=R_{0} e^{\frac{\left(2 p_{0}-Y_{j}^{\text {ini }}\right) /\left(1+\xi_{j}^{\text {ini }}\right)-\sigma_{0}}{Y_{j}^{\text {res }}}} .
$$

For the deep underground engineering, the real displacement far away from the tunnel surface is almost zero after the tunnel excavation. Hence, the initial displacement values, that is, $\left(\left(1-v-2 v^{2}\right) p_{0} r / E\right)$, caused by the hydrostatic pressure should be ignored at the infinite boundary $\left(R_{2} \longrightarrow \infty\right)$. Therefore, the radial displacement caused by tunnel excavation in the elastic zone should be rewritten as follows:

$$
u_{r(2)}=\frac{(1+v)\left(p_{0}-\sigma_{R_{1}}\right)}{E}\left(\frac{R_{1}^{2}}{r}\right) .
$$

The radial displacement at the elastic-plastic interface can be determined by equations (64a) and (64b) as follows:

$$
u_{R_{1}}=\frac{(1+v)\left(p_{0}-\sigma_{R_{1}}\right)}{E} R_{1} .
$$

Just as the calculation method of radial displacement in Section 4.2, four different assumptions about the elastic strain forms are also used to research the deformation behavior of rock mass in the plastic zone.

(1) Case 1: with the assumption of the constant elastic strain at the elastic-plastic interface, as the calculation methods in equations (45) and (46), the function $f(r)$ can be easily deduced by integrating equations (65) and (32) as follows:

$$
f(r)=-\frac{(1+\nu)(1-\beta)}{E}\left(p_{0}-\sigma_{R_{1}}\right)=\delta_{\text {casel }} \text {. }
$$

Then, the radial displacement of plastic zone for deep circular opening will be obtained by introducing equations (67) and (66) into equation (43). The expression form is consistent with equation (47) except for the parameters $\delta_{\text {casel }}$ (taking equation (67)) and $u_{R_{1}}$ (taking equation (66)).

(2) Case 2: as shown in equations (49) and (50), the radial displacement for deep circular opening can be also obtained by regarding the plastic zone as the thick-wall cylinder. The expression is the same as that in equation (50) except for $u_{R_{1}}$ (equation (66)) and $\sigma_{R_{1}}$ (equation (63)).

(3) Case 3: by adopting generalized Hooke's law for removing the effect of initial hydrostatic pressure $p_{0}$, the elastic strain in the plastic zone can be expressed as follows:

$$
\left\{\begin{array}{l}
\varepsilon_{\theta(1)}^{e}=\frac{\left(1+v_{\text {res }}\right)}{E_{\text {res }}}\left[\left(1-v_{\text {res }}\right)\left(\sigma_{\theta(1)}-p_{0}\right)-v_{\text {res }}\left(\sigma_{r(1)}-p_{0}\right)\right] . \\
\varepsilon_{r(1)}^{e}=\frac{\left(1+v_{\text {res }}\right)}{E_{\text {res }}}\left[\left(1-v_{\text {res }}\right)\left(\sigma_{r(1)}-p_{0}\right)-v_{\text {res }}\left(\sigma_{\theta(1)}-p_{0}\right)\right] .
\end{array}\right.
$$

Then, the function $f(r)$ can be expressed as follows:

$$
\begin{aligned}
f(r)= & \left(\frac{1+v_{\text {res }}}{E_{\text {res }}}\right)\left[\left(1-v_{\text {res }}-\beta v_{\text {res }}\right) \sigma_{r(1)}\right. \\
& \left.+\left(\beta-v_{\text {res }}-\beta v_{r e s}\right) \sigma_{\theta(1)}+\left(2 v_{\text {res }}-1\right)(1+\beta) p_{0}\right] .
\end{aligned}
$$


(i) If $\xi_{j}^{\text {res }} \neq 1$, the radial displacement for deep circular opening can be deduced by calculation methods similar to those discussed in Section 4.1. Its expression is the same as that in equation (54), but the constant $\delta_{2}^{\text {case3 }}$ in equation (54) needs to be replaced by

$$
\delta_{22}^{\text {case3 }}=\left(1-2 v_{\text {res }}\right)(1+\beta)\left[\frac{p_{0}-Y_{j}^{\text {res }}}{\left(\xi_{j}^{\mathrm{res}}-1\right)}\right] .
$$

(ii) If $\xi_{j}^{\text {res }}=1$, the radial displacement for TR and VM criteria can be also obtained by substituting equations (38a), (38b), and (69) into equation (43). The expression is in accordance with equation (56); after that the constant $\delta_{4}^{\text {case3 }}$ in equation (56) is replaced by

$$
\begin{aligned}
\delta_{44}^{\text {case3 }}= & \left(1-v_{\text {res }}-\beta v_{\text {res }}\right) \sigma_{0}+\left(\beta-\nu_{\text {res }}-\beta \nu_{\text {res }}\right)\left(\sigma_{0}+Y_{j}^{\text {res }}\right) \\
& +\left(2 v_{\text {res }}-1\right)(1+\beta) p_{0} .
\end{aligned}
$$

(4) Case 4: in this case, the influence of rock mass damage degree on the mechanical and deformation behavior of surrounding rock is taken into account by assuming Young's modulus attenuation along the radius direction. As discussed in Section 4.1, for $\xi_{j}^{\text {res }} \neq 1$, the radial displacement for deep circular opening in the plastic zone can be determined through the constant $\delta_{2}^{\text {case3 }}$ in equation (59) replaced by the value $\delta_{22}^{\text {case } 3}$ in equation (70). In addition, for $\xi_{j}^{\text {res }}=1$, the radial displacement based on TR and VM criteria will be calculated by the constant $\delta_{4}^{\text {case3 }}$ in equation (61) replaced by the value $\delta_{44}^{\text {case } 3}$ in equation (71).

\section{The Theoretical and Practical Value}

The new unified closed-form solution obtained in Section 4.1 with a finite boundary can be regarded as a semianalytical solution due to solving equations (37) and (39) by a numerical calculation. In addition, the new closed-form solution for a deep circular opening in Section 4.2 with an infinite boundary is a true analytical solution. The new closed-form solution with the infinite and finite external boundary can be used to predict the stress and displacement of circular opening excavated in an elastic-brittle plastic rock mass under different yield criteria. Actually, the circular opening maybe includes a borehole, vertical shaft, and deep- or shallow-buried tunnel. In addition, the new unified solution proposed in this paper is a series of traditional solutions rather than one specific solution. The solution based on the MC, GMC, TR, VM, GLD, GSMP, DP (CDP, MDP, and IDP), UST, and GTSS yield criteria can be obtained from the new unified solution by making $\xi_{j}$ and $Y_{j}$ take different parameters (as shown in Table 1). Therefore, the new unified solution has a broader engineering application scope and can be more appropriate for different lithology rock masses.
Zhang et al. [6], Park and Kim [9], and Chen et al. [17] carried out relevant theoretical research on the new closedform solution of circular opening as discussed in Sections 4.1 and 4.2. However, the solution by Zhang et al. was based on the unified strength theory and ignored the influence of the other criteria on the mechanical response of rock mass. Hence, the application of this solution has some limitations for different lithology rock masses. Chen's solution, based on the elastic, perfectly plastic model and Mohr-Coulomb criterion, did not reflect the effect of internal principal stress and strength parameter attenuation. In addition, Park and Kim [9] analyzed the deformation law of plastic zone by using three different definitions for elastic strains in the plastic zone with Mohr-Coulomb (MC) and Hoke-Brown (HB) criteria. However, the solution based on the finite external boundary was not given. For the shallow-buried tunnel, it may not be reasonable to regard the outer boundary of circular tunnel as the infinite boundary. Moreover, the internal principal stress and Young's modulus attenuation are also ignored in this paper. When $j=$ UST, the new unified solution proposed by this paper will be converted to the results by Zhang et al. [6] except for Case 1 and Case 2. When $j=\operatorname{MC} v_{\text {res }}=v, c_{\text {res }}=c, \varphi_{\text {res }}=\varphi$, and $E_{\text {res }}=E$, the solution based on this paper will be transformed into the solution by Chen et al. [17]. Meanwhile, Park and Kim's solution based on MC criterion is a special case in Section 4.2 of this paper except for Case 4. Therefore, the new unified closed-form solution obtained in this paper is a series of results, and it is suitable for a wide range of rock masses and engineering backgrounds. Meanwhile, it can provide a broader yield criterion for the engineering application as well.

\section{Correctness Verification and Parameter Analysis}

6.1. A Comparison with the Traditional Solution. Park et al. summarized three different definitions for elastic strains in the plastic zone (see Cases 1 3 in Section 4.2) and analyzed the deformation law of plastic zone with Mohr-Coulomb criterion [9]. However, the Young's modulus and Poisson's ratio attenuation were ignored. In fact, it can be obtained by taking $E_{\text {res }}=E, \quad \nu_{\text {res }}=v, \quad \xi_{j}=\xi_{\mathrm{MC}}$, and $Y_{j}=Y_{\mathrm{MC}}$ in this paper. To further verify the correctness of the calculation results for deep circular opening in Section 4.2, the solution by Park et al. will be presented as a comparison with the solution proposed by this paper. The geometrical and physical parameters for circular opening are shown in Table 2.

The radial displacements obtained by Park are shown in Figure 7 in this paper. It can be seen that the calculation results in this paper are in accordance with Park's closedform solution. Therefore, the closed-form solution proposed by this paper is correct and can be regarded as an extension of Park's solution. In other words, this paper's solution has a more wide application in practice engineering. 
TABLE 2: Geometrical and physical parameters of circular opening.

Parameters

Rock type by Park and Kim (2006) [10]

Radius of opening, $R_{0}(\mathrm{~m})$

Initial stress, $\sigma_{0}(\mathrm{MPa})$

Internal pressure, $p_{\text {in }}(\mathrm{MPa})$

Young's modulus, $E(\mathrm{MPa})$

Poisson's ratio, $v$

Shear modulus, $G(\mathrm{MPa})$

$c(\mathrm{MPa})$

$\varphi$ (deg)

$c_{\text {res }}(\mathrm{MPa})$

$\varphi_{\text {res }}(\mathrm{deg})$

Hard rock

Soft rock
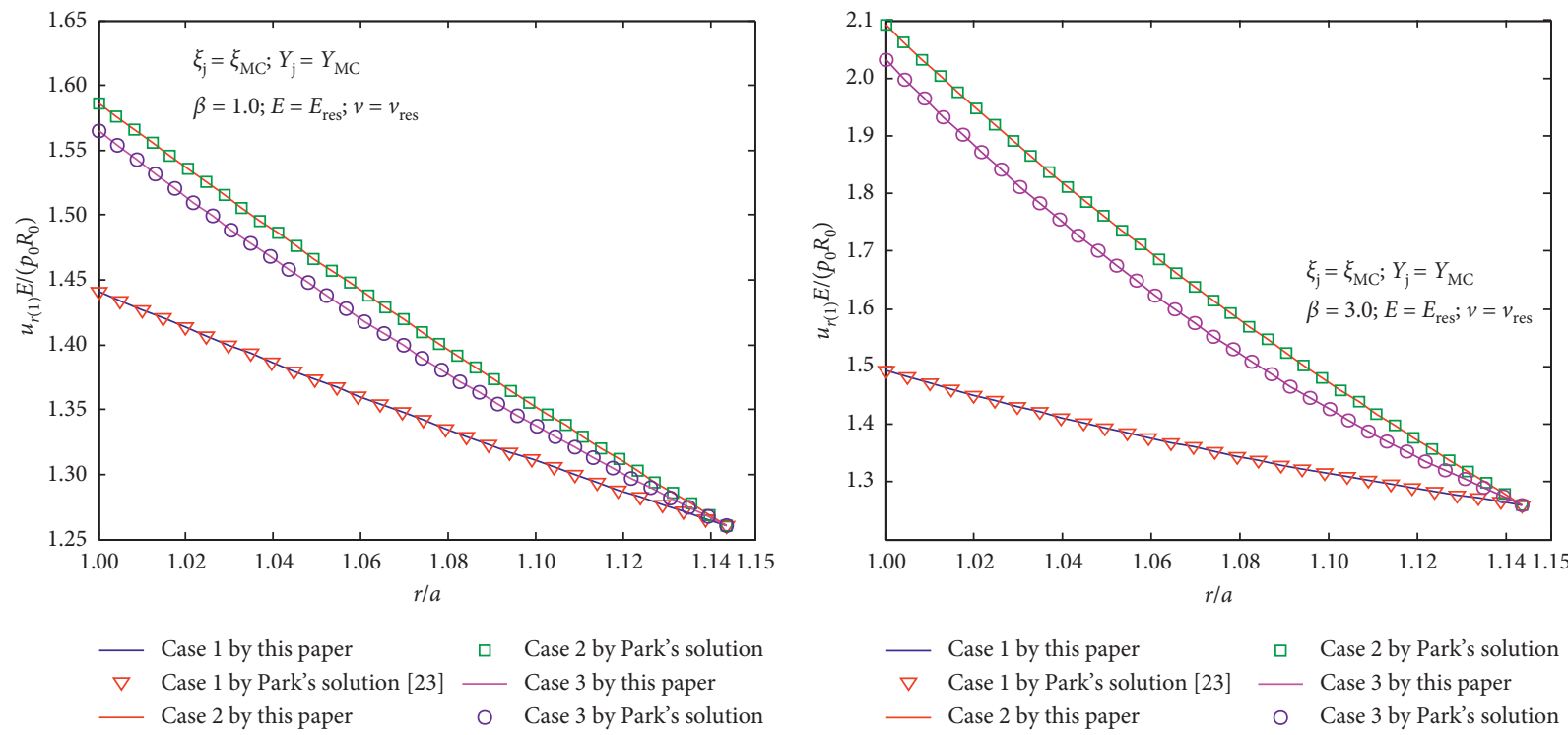

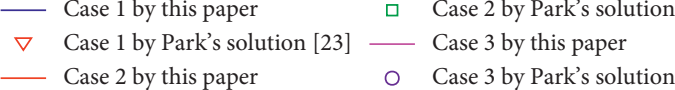

(a)

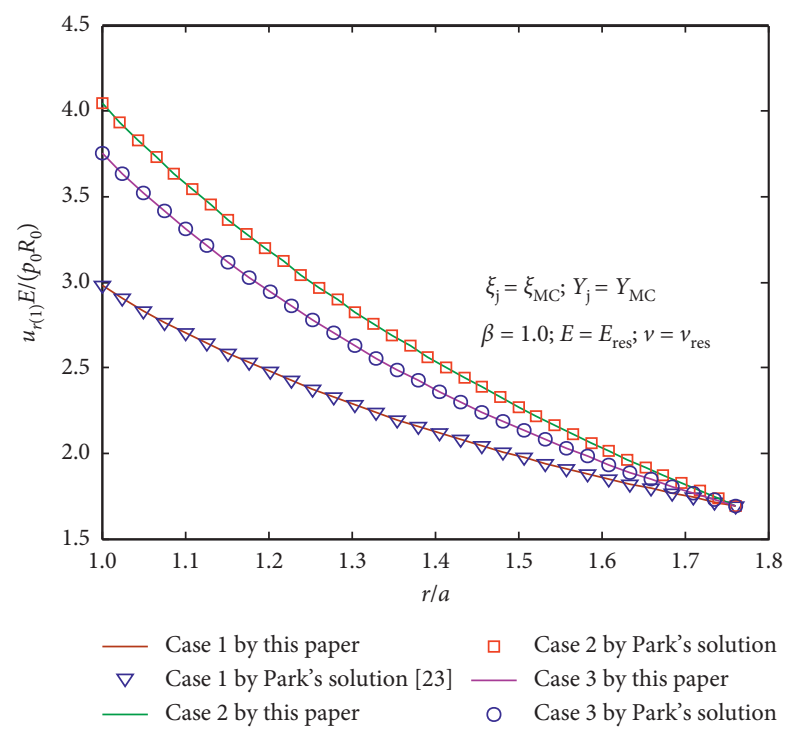

(c)

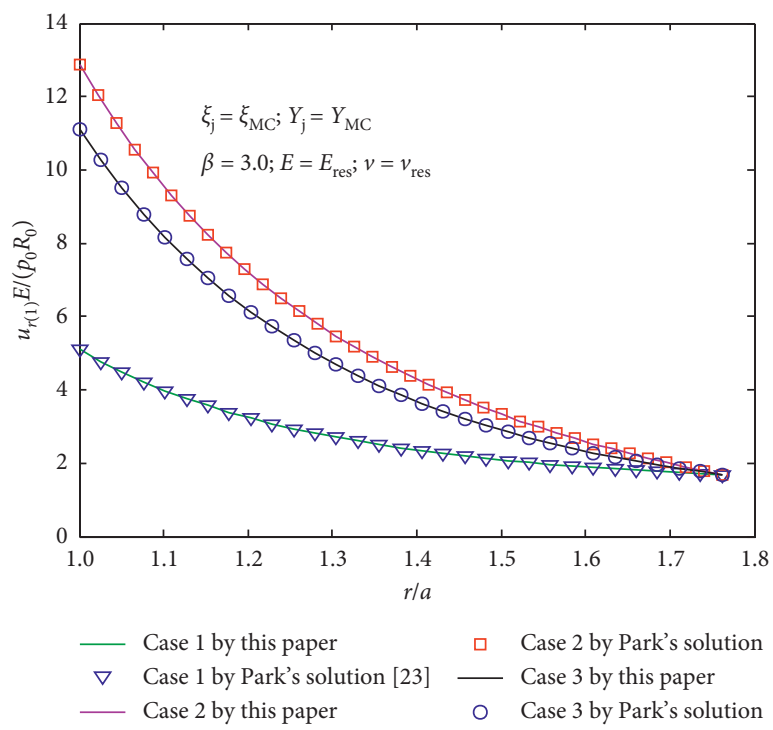

(d)

Figure 7: Comparison with the traditional solution. (a) Radial displacement (data: hard rock, $\beta=1.0$ ). (b) Radial displacement (data: hard rock, $\beta=3.0$ ). (c) Radial displacement (data: soft rock, $\beta=1.0$ ). (d) Radial displacement (data: soft rock, $\beta=3.0$ ). 


\subsection{Parameters Analysis}

6.2.1. The Effect of the Strength Yield Criteria. As previously mentioned, the strength criteria exert an extremely important effect for evaluating the mechanical response and deformation behavior of surrounding rock. For studying the influence of strength theories on the stresses and displacement of surrounding rock, the mechanical and geometrical parameters of circular tunnel are shown in Table 3.

The stresses distribution laws under different strength criteria are shown in Figure 8. Meanwhile, the dimensionless radius of plastic zone and critical inner pressure at the elastic-plastic interface are presented in Table 4 . It can be seen that the dimensionless values $\left(\left(R_{1} / R_{0}\right)\right.$ and $\left.\left(\sigma_{R_{1}} / p_{0}\right)\right)$ show the characteristics of $\mathrm{TR}>\mathrm{VM}>\mathrm{IDP}>\mathrm{MC}>$ MDP $>$ UST0.5 > GSMP > GMC > GLD > GTSS > CDP. Compared with the traditional solution obtained by MC criterion, the dimensionless value $\left(\left(R_{1} / R_{0}\right)\right)$ calculated by TR and VM criteria obviously increases by $125.26 \%$ and $78.53 \%$, respectively. This is mainly because the TR and VM criteria only regard the rock mass as friction-less bonding material and ignore the impact of friction effect on the mechanical properties of the rock mass so that the bearing capacity of rock mass is underestimated. Therefore, the calculation result is obviously larger than the solution obtained by other criteria. The MC criterion does not take the effect of the intermediate principal stress into account and it is easy to underestimate the bearing capacity of rock mass. Therefore, the deformation of surrounding rock calculated by MC criterion may be slightly larger. The IDP and MDP criteria underestimate the influence of internal principal stresses, so the calculation results may also be larger than the solution obtained by UST0.5, GSMP, GMC, GLD, CDP, and GTSS criteria. Meanwhile, the result obtained by CDP criterion is minimal compared with other criteria's solutions. In fact, the criterion overestimates the effect of intermediate principal stresses on rock mass strength and may not be reasonable in practical engineering. In addition, the calculation results $\left(\left(R_{1} / R_{0}\right)\right)$ obtained by UST0.5, GSMP, GMC, and GLD criteria are close to each other within the range of 1.294-1.347. The above four criteria seem to be more reasonable considering the effect of intermediate principal stress on yield strength of rock masses.

From the above analysis, it can be seen that the UST0.5, GSMP, GMC, and GLD criteria can be strongly recommended for evaluating the mechanics and deformation behavior of surrounding rock, followed by IDP, MDP, GTSS, and MC criteria. The TR, VM, and CDP criteria are not recommended to be used for underground engineering.

6.2.2. The Effect of Dilation Coefficient. As shown in Figure 9, the dilation coefficient has an extremely important influence on the surface displacement of surrounding rock. As the parameter $\beta$ gradually increases, the dimensionless surface displacement $\left(u_{0} E /\left(p_{0} R_{0}\right)\right)$ presents the nonlinear increase characteristics. However, the increasing rate of surface displacement under different yield criteria is significantly different. For instance, as shown in Table $5, \beta$ increases from 1.0 to 3.0 , and the dimensionless value $\left(u_{0} E /\left(p_{0} R_{0}\right)\right)$, respectively, increases by $38.12 \%$ for MC criterion, $16.94 \%$ for GSMP criterion, $18.30 \%$ for UST0.5 criterion, and $29.58 \%$ for MDP criterion under Case 1 condition. Therefore, the effect of dilation coefficient should be considered in the design of tunnel support parameters and strength.

6.2.3. The Effect of Elastic Strain Form and Rock Mass Damage. In this study, the geometrical and mechanical parameters of circular tunnel are shown in Table 3 (soft rock). Figure 10 presents the influence of elastic strain form in the plastic zone on the surface displacement of surrounding rock. From the above analysis, the relevant conclusions can be summarized as follows:

(i) The radial displacement of plastic zone is closely related to the selection of the elastic strain form. Case 2 has the greatest effect on the radial displacement of plastic zone, followed by Case 3. Then, the results obtained by Case 2 are minimal. For example, when $r=R_{0}$ (tunnel surface), compared with Case 1 , the dimensionless surface displacement $\left(u_{0} E /\left(p_{0} R_{0}\right)\right)$, respectively, increases by 0.272 for Case 2 and 0.206 for Case 3 with an increasing rate of $16.47 \%$ for Case 2 and $12.46 \%$ for Case 3 under GLD criterion.

(ii) Meanwhile, the influence of elastic strain form on the radial displacement of plastic zone is also closely related to the strength criterion. For example, the dimensionless surface displacements $u_{0} E /\left(p_{0} R_{0}\right)$ are, respectively, 1.926 for Case 2 and 1.860 for Case 3 under GLD criterion. However, the value significantly increases by $18.32 \%$ for Case 2 and $31.71 \%$ for Case 3 under GTSS criterion.

When the rock mass enters the plastic zone, its mechanical and deformation behavior are closely related to the rock damage degree. Generally, Young's modulus attenuation could be used to indicate the damage degree of rock mass. The influence of rock mass damage degree on the surface displacement of surrounding rock is shown in Figure 11.

(iii) It can be seen that the radial displacement of plastic zone is closely related to the selection of Young's modulus attenuation model. Compared with Case 3 $\left(E_{\text {res }}=E\right)$, Case 4 has the greatest effect on the radial displacement of plastic zone. Case 3 $\left(E_{\text {res }}=0.65 E\right)$ is the second. For example, as shown in Table 6, compared with Case $3\left(E_{\text {res }}=E\right)$, the dimensionless surface displacements $\left(u_{0} E /\left(p_{0} R_{0}\right)\right)$ of Case $3\left(E_{\text {res }}=0.65 E\right)$ and Case 4 increase by 0.163 and 0.074 with an increasing rate of $7.49 \%$ and $3.40 \%$, respectively, under the GTSS criterion.

(iv) In addition, the influence of Young's modulus attenuation on the radial displacement of plastic zone is closely related to the strength criterion. For Case 3 $\left(E_{\text {res }}=0.65 E\right)$ and Case 4 , the dimensionless surface displacements $\left(u_{0} E /\left(p_{0} R_{0}\right)\right)$ of surrounding rock are 1.971 and 1.911 , respectively, under the 
TABLE 3: The mechanical and geometrical parameters of circular opening.

\begin{tabular}{cccccccc}
\hline$p_{0}(\mathrm{MPa})$ & $\sigma_{0}(\mathrm{MPa})$ & $R_{0}(\mathrm{~m})$ & $E_{\text {res }}=E(\mathrm{GPa})$ & $v_{\text {res }}=\nu$ & $c_{\text {res }}=c(\mathrm{MPa})$ & $\varphi_{\text {res }}=\varphi\left({ }^{\circ}\right)$ & $\beta$ \\
\hline 25 & 0 & 3 & 3 & 0.25 & 7.2 & 18.3 & 1.0 \\
\hline
\end{tabular}

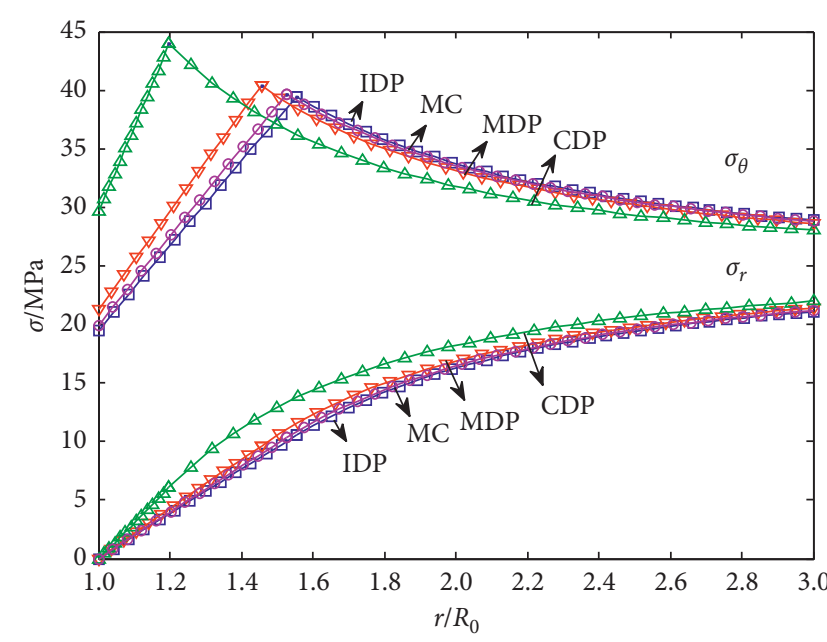

(a)

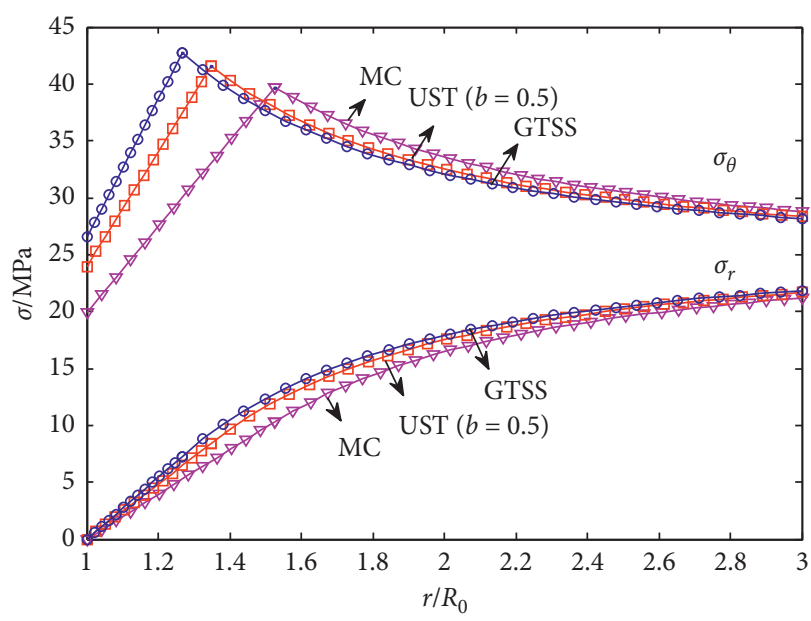

(c)

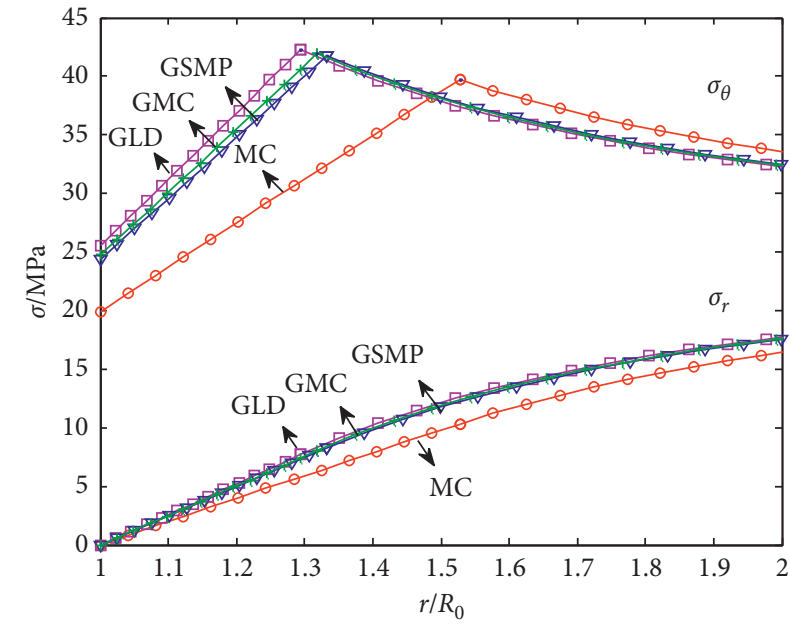

(b)

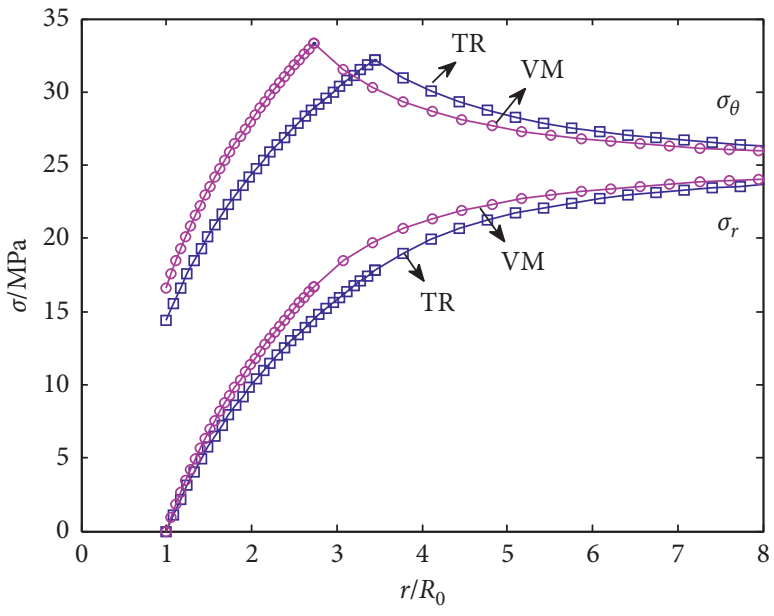

(d)

FIGURE 8: The stresses distributions law under different criteria.

TABLE 4: The radius of plastic zone and critical inner pressures under different criteria.

\begin{tabular}{lccc}
\hline Strength criterion & $\left(R_{1} / R_{0}\right)\left(\sigma_{R_{1}} / p_{0}\right)$ & Strength criterion & $\left(R_{1} / R_{0}\right)\left(\sigma_{R_{1}} / p_{0}\right)$ \\
\hline MC & $1.528(0.413)$ & VM & $2.728(0.667)$ \\
GMC & $1.318(0.322)$ & IDP & $1.556(0.422)$ \\
GLD & $1.294(0.308)$ & MDP & $1.456(0.386)$ \\
GSMP & $1.332(0.329)$ & CDP & $1.198(0.242)$ \\
TR & $3.442(0.712)$ & UST $_{0.5}$ & $1.347(0.337)$ \\
GTSS & $1.266(0.291)$ & - & - \\
\hline
\end{tabular}

GLD criterion. However, when taking the GSMP criterion, the value significantly increases by $25.82 \%$ and $24.54 \%$ compared with the GLD criterion, respectively.
Figure 12 illustrates the effect of elastic strain form on the ground response curve. From Figure 12, it can be seen that the surface displacement of surrounding rock presents the change characteristic of Case 2 solution $>$ Case 3 


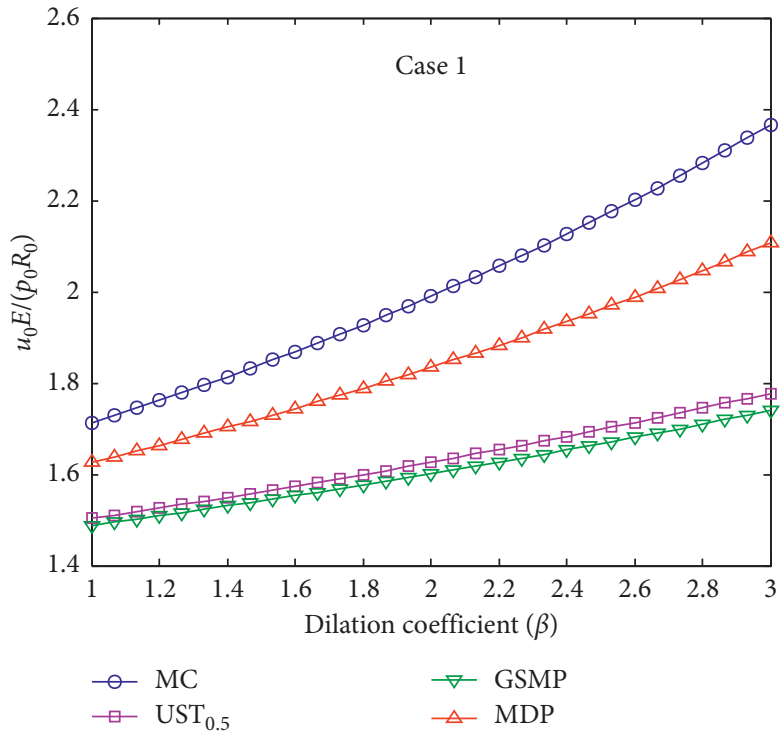

(a)

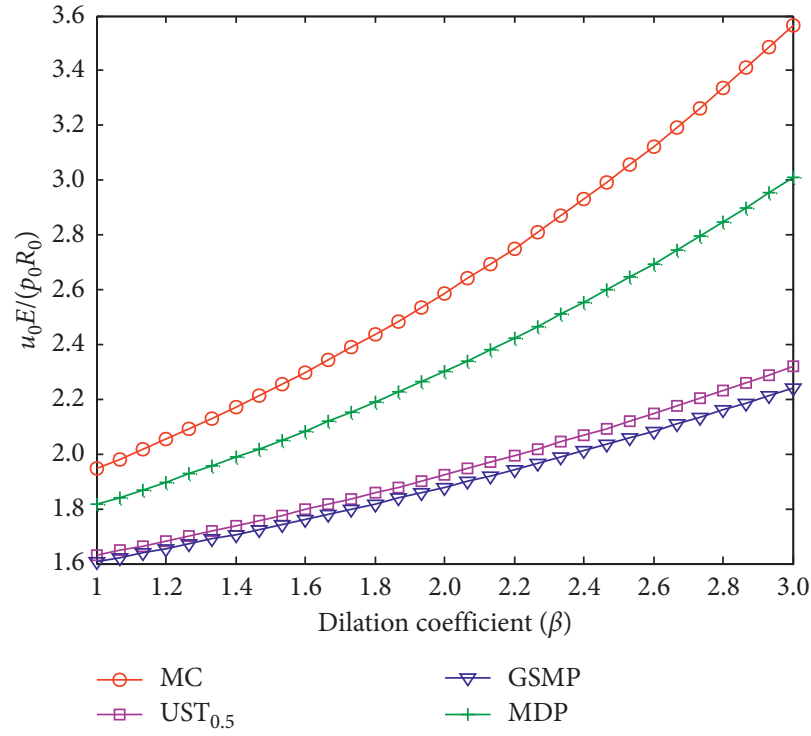

(b)

Figure 9: The effect of dilation coefficient on the surface displacement.

TABLE 5: The surface displacement value $\left(u_{0} E /\left(p_{0} R_{0}\right)\right)$ of the circular tunnel.

\begin{tabular}{|c|c|c|c|c|c|}
\hline & Parameter $(\beta)$ & $\mathrm{MC}$ & GSMP & $\mathrm{UST}_{0.5}$ & MDP \\
\hline \multirow{3}{*}{ Case 1} & 1.0 & 1.713 & 1.488 & 1.503 & 1.626 \\
\hline & 2.0 & 1.990 & 1.601 & 1.626 & 1.834 \\
\hline & 3.0 & 2.366 & 1.740 & 1.778 & 2.107 \\
\hline \multirow{3}{*}{ Case 2} & 1.0 & 1.945 & 1.607 & 1.630 & 1.815 \\
\hline & 2.0 & 2.5867 & 1.879 & 1.924 & 2.302 \\
\hline & 3.0 & 3.567 & 2.241 & 2.320 & 3.011 \\
\hline
\end{tabular}

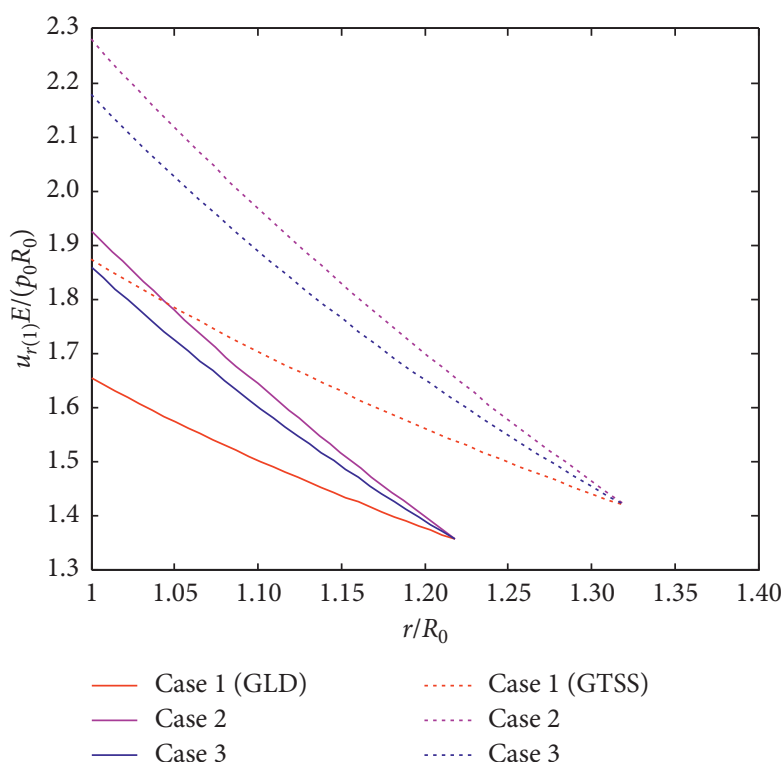

(a)

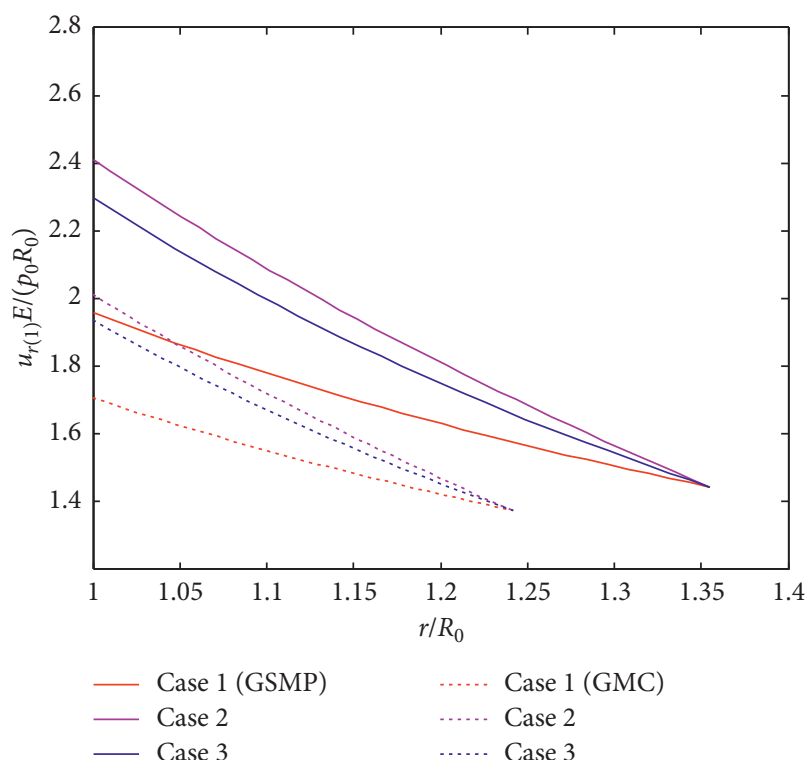

(b)

Figure 10: The effect of elastic strain form on the radial displacement. 


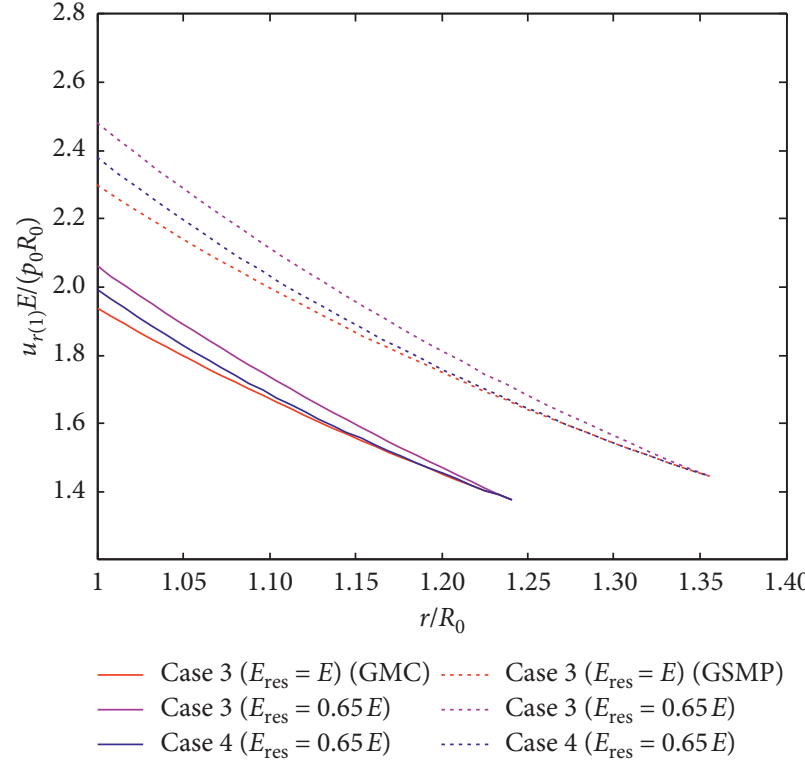

(a)

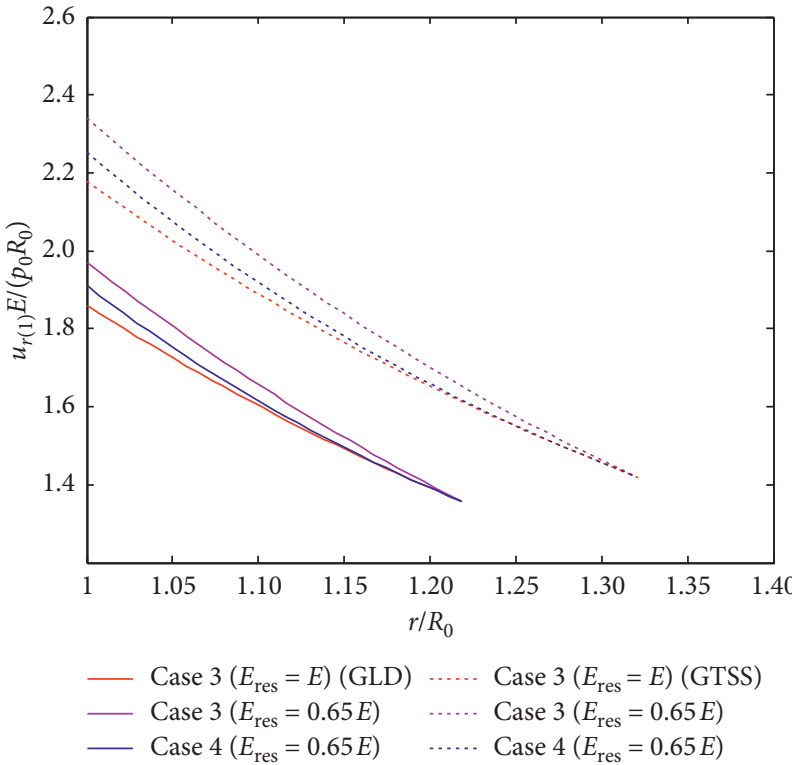

(b)

FIgURE 11: The effect of rock mass damage on the radial displacement.

TABle 6: The dimensionless surface displacement $\left(u_{0} E /\left(p_{0} R_{0}\right)\right)$ of the circular tunnel.

\begin{tabular}{llllll}
\hline & & GLD & GTSS & GMC & GSMP \\
\hline Case 3 & $m=0, E_{\text {res }}=E$ & 1.860 & 2.177 & 1.937 & 2.297 \\
Case 4 & $m \neq 0, E_{\text {res }}=0.65 E$ & 1.971 & 2.340 & 2.061 & 2.480 \\
& $m \neq 0, E_{\text {res }}=0.65 E$ & 1.911 & 2.251 & 1.993 & 2.380 \\
\hline
\end{tabular}

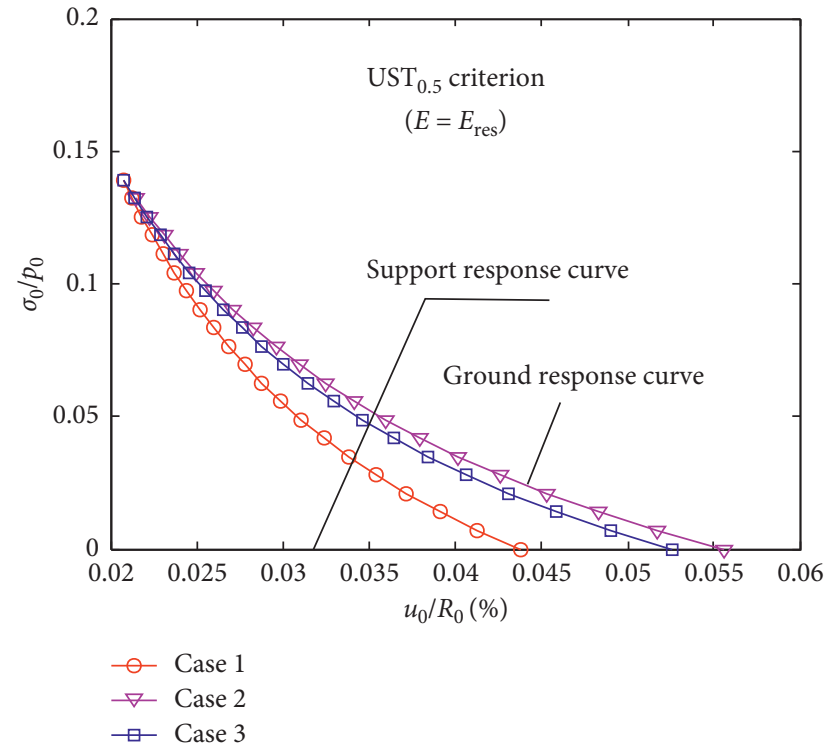

(a)

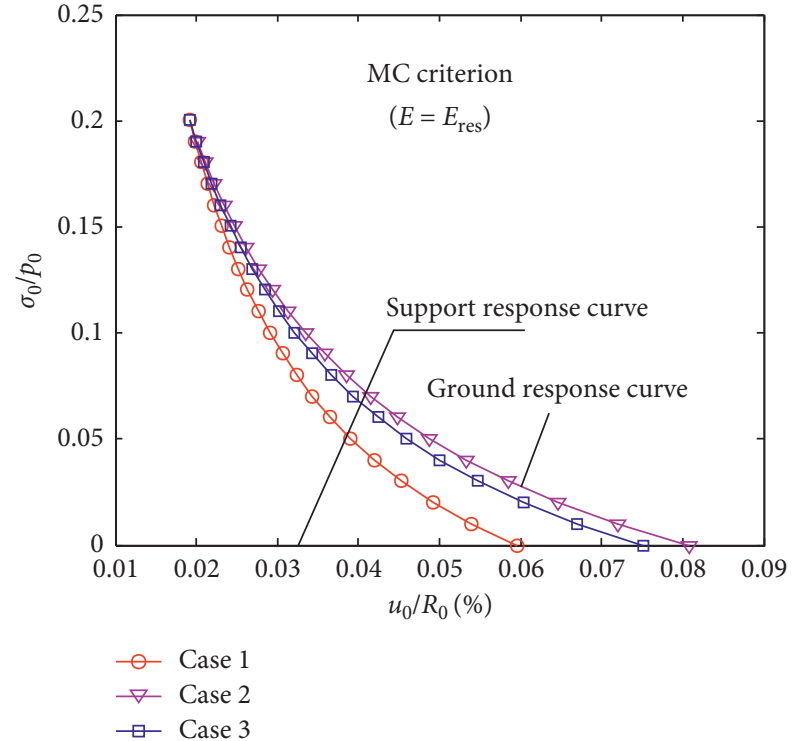

(b)

Figure 12: Continued. 


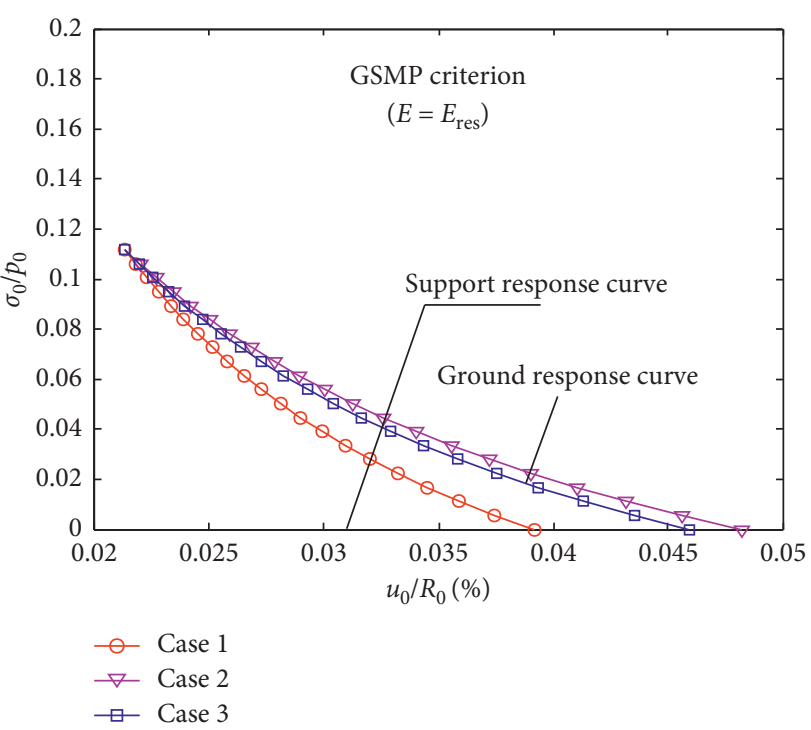

(c)

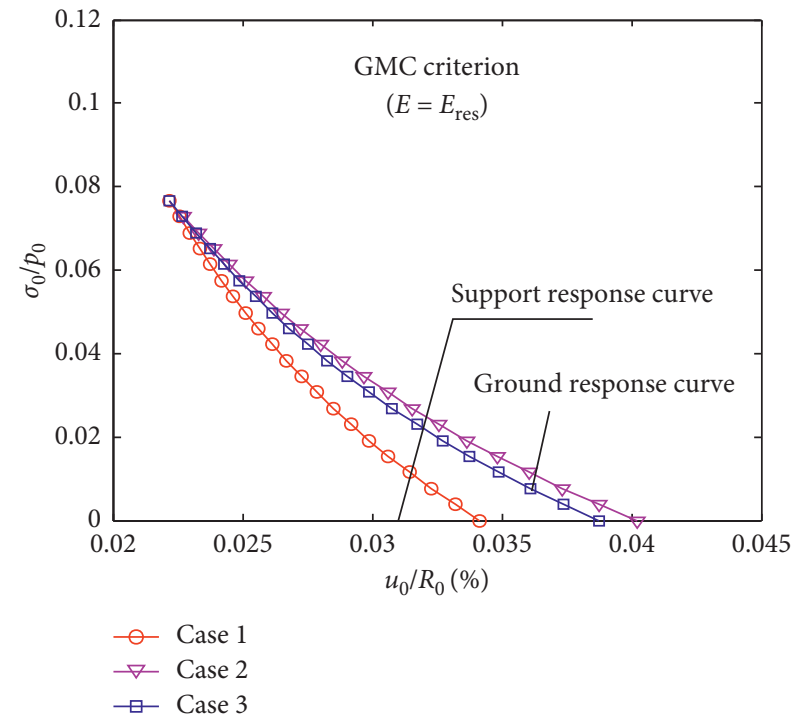

(d)

Figure 12: The effect of elastic strain form on the ground response curve (note: Young's modulus attenuation is ignored in Case 3).

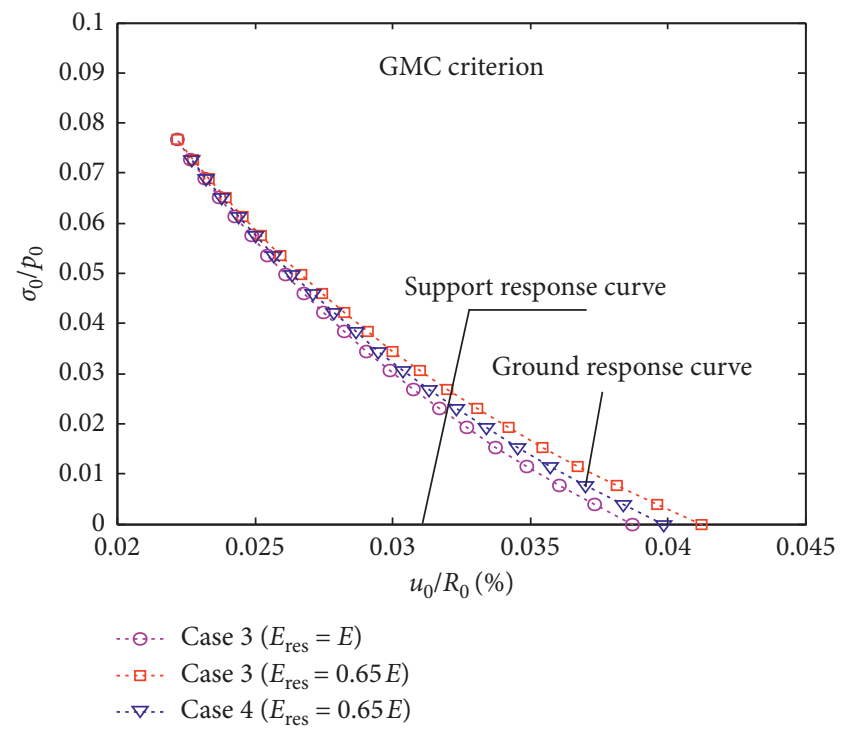

(a)

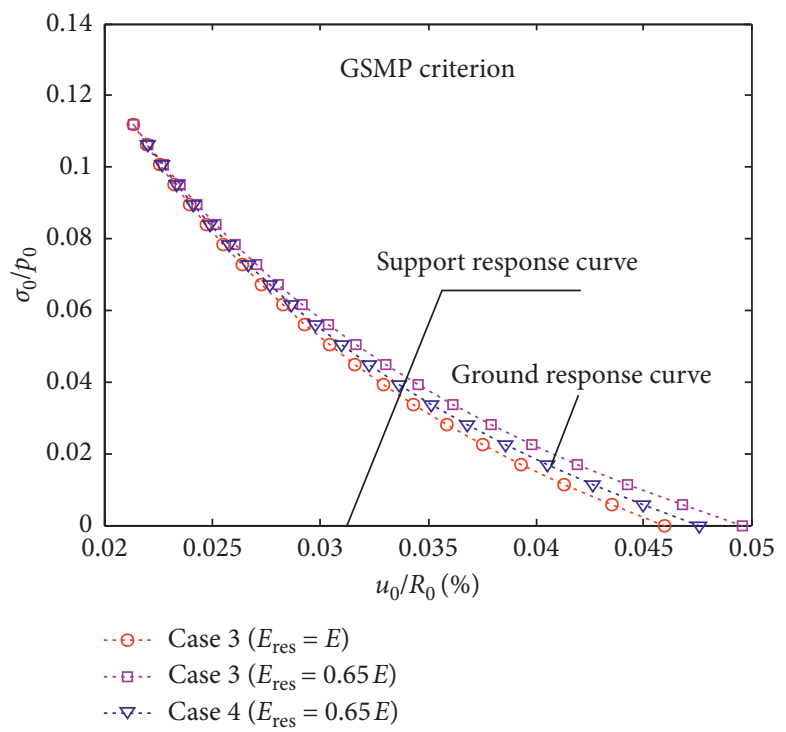

(b)

FIGURE 13: The effect of rock mass damage on the ground response curve.

solution $>$ Case 1 solution when the support pressures are equal under the same strength criterion. For example, when $\left(\sigma_{0} / p_{0}\right)=0$, compared with Case 2, the surface displacements of surrounding rock decrease, respectively, by $26.37 \%$ for Case 1 and $7.13 \%$ for Case 3 under the MC strength criterion or by $18.84 \%$ for Case 1 and $4.76 \%$ for Case 3 under the GSMP strength criterion. From the above analysis, we can see that the support strength design should satisfy the relationship of Case $2>$ Case $3>$ Case 1 under the same deformation conditions. In fact, the support strength predicted by using Case 2 is relatively large, while that predicted by using Case 1 is relatively small.
In addition, the change characteristic of the ground response curve is closely related to the section of strength criterion. For instance, when $\left(\sigma_{0} / p_{0}\right)=0$, the surface displacement $\left(\left(u_{0} / R_{0}\right)\right)$ increases, respectively, by $74.46 \%$ for the MC criterion, $14.68 \%$ for GSMP criterion, and $28.17 \%$ for UST 0.5 criterion by comparison with the GMC criterion under Case 1 condition. The main reason for the above results is that the different strength criteria are different in evaluating the bearing capacity of rock mass. For example, the $\mathrm{MC}$ criterion does not consider the effect of intermediate principal stress and then it is easy to underestimate the bearing capacity of rock mass. Therefore, the surface 
displacement obtained by MC criterion is larger than that obtained by other criteria under the same support pressure.

The ground and support response curves under GMC and GSMP criteria are shown in Figure 13 considering different Young's modulus attenuation models. It is shown that the damage degree of rock mass has an extremely important influence on the ground response curve. For instance, when $\sigma_{0} / p_{0}=0$, compared with Case $3\left(E_{\text {res }}=E\right)$, the surface displacements of surrounding rock increase, respectively, by $7.96 \%$ for Case $3\left(E_{\text {res }}=0.65 E\right)$ and $3.59 \%$ for Case 4 under GSMP criterion. In other words, when the Young's modulus attenuation is ignored (Case $3\left(E_{\text {res }}=E\right)$ ), the deformation of the surrounding rock and support force will be underestimated; however, when a residual value of Young's modulus is assumed in the plastic zone (Case 3 $\left(E_{\text {res }}=0.65 E\right)$ ), the deformation of the surrounding rock and support force will be overestimated. So, the Young's modulus power function attenuation model (Case 4) seems to present more reasonable results and is recommended for optimizing support parameters design and evaluating stability of surrounding rock in deep underground engineering.

\section{Conclusions}

By summarizing and simplifying different strength theories, a new unified criterion equation is firstly proposed based on the certain assumption. Then, a new unified closed-form solution for circular opening based on the newly proposed unified criterion equation is deduced with the infinite and finite boundary combining with the nonassociative flow rule. In the plastic zone, four different elastic strain assumptions are applied to solving the plastic zone deformation considering the effect of rock mass damage. Then, the validity of the solution is also verified by comparison with the traditional solution. Finally, the influences of strength criterion effect, dilation coefficient, elastic strain form, and rock mass damage on the mechanical response of surrounding rock are discussed in detail. The primary conclusions can be summarized as follows:

(1) The selection of strength criterion has an extremely important effect on the stresses, plastic zone radius, and critical inner pressure distributions of surrounding rock. For the radius of plastic zone, the calculation results obtained by TR and VM criteria which ignore the impact of friction effect are obviously the largest, followed by IDP, MC, and MDP criteria. The result obtained by CDP criterion which overestimates the effect of intermediate principal stresses on rock mass strength is minimal compared with other criteria's solutions. In addition, the calculation results obtained by $\mathrm{UST}_{0.5}$, GSMP, GMC, and GLD criteria which reasonably consider the effect of internal principal stresses give an intermediate range. Therefore, $\mathrm{UST}_{0.5}$, GSMP, GMC, and GLD criteria can be strongly recommended for evaluating the mechanics and deformation behavior of surrounding rock, followed by IDP, MDP, GTSS, and MC criteria. TR, VM, and CDP criteria are not recommended to be used.
(2) The influence of dilation coefficient on the surface displacement of surrounding rock is closely related not only to the assumption form of elastic strain in the plastic zone but also to the strength criteria. As the dilation coefficient gradually increases, the dimensionless surface displacement presents the nonlinear increase characteristics. Therefore, the support parameters design should take the influence of the dilation coefficient into account.

(3) The elastic strain assumption forms in the plastic zone have a significant important effect on the deformation of plastic zone and ground response curve by ignoring the effect of rock mass damage. Case 1 gives a smallest deformation and seems to overestimate the plastic bearing capacity of rock mass; however, Case 2 gives a largest deformation and maybe underestimates the plastic bearing capacity of rock mass. Therefore, Case 3 may be more reasonable for evaluating stability of surrounding rock and optimizing support strength design.

(4) The effects of Young's modulus attenuation on the deformation of the plastic zone and ground response curve are related to the selection of the Young's modulus attenuation model and also closely related to the strength criterion. When ignoring the continuity of Young's modulus attenuation, the deformation of surrounding rock is easy to be overestimated or underestimated. So, the Young's modulus power function attenuation seems to give more reasonable results.

\section{Data Availability}

Data supporting the results of this study are included in the article.

\section{Conflicts of Interest}

The authors declare that there are no conflicts of interest regarding the publication of this paper.

\section{Acknowledgments}

This work was financially supported by the Fundamental Research Funds for the Central Universities (2017XKQY097).

\section{References}

[1] T. Ogawa and K. Y. Lo, "Effects of dilation and yield criterion on displacement around tunnels," Canadian Geotechnical Journal, vol. 24, no. 1, pp. 100-113, 1987.

[2] A. M. Al-Ajmi and R. W. Zimmerman, "Stability analysis of vertical boreholes using the Mogi-Coulomb failure criterion," International Journal of Rock Mechanics \& Mining Sciences, vol. 43, no. 8, pp. 1200-1211, 2006.

[3] X. J. Wang, M. X. Chen, X Chang et al., "Studies of application of Drucker-Prager yield criterion to stability analysis," Rock and Soil Mechanics, vol. 30, no. 12, pp. 3733-3738, 2009. 
[4] C. G. Zhang, C. L. Zhang, F. Zhou et al., "The strength theory effect in elastic-plastic analysis of a circular tunnel," Chinese Journal of Geotechnical Engineering, vol. 20, no. 10, pp. 1-8, 2017.

[5] L. Chen, X. B. Mao, Y. L. Chen et al., "A new unified solution for circular tunnel based on a four-stage constitutive model considering the intermediate principal stress," Advances in Civil Engineering, vol. 2018, p. 14, 2018.

[6] C. Zhang, J. Zhao, Q. Zhang et al., "A new closed-form solution for circular openings modeled by the unified strength theory and radius-dependent young's modulus," Computers \& Geotechnics, vol. 42, no. 42, pp. 118-128, 2012.

[7] C. Carranza-Torres, "Elasto-plastic solution of tunnel problem using the generalized form of the Hoek-Brown failure criterion," International Journal of Rock Mechanics and Mining Sciences, vol. 41, no. 3, pp. 480-1, 2004.

[8] D. Ma, M. Rezania, H. S. Yu, and H. B. Bai, "Variations of hydraulic properties of granular sandstones during water inrush: effect of small particle migration," Engineering $\mathrm{Ge}$ ology, vol. 217, pp. 61-70, 2017.

[9] S. K. Sharan, "Exact and approximate solutions for displacements around circular openings in elastic-brittle-plastic Hoek-Brown rock," International Journal of Rock Mechanics and Mining Sciences, vol. 42, no. 4, pp. 542-549, 2005.

[10] K. H. Park and Y. J. Kim, "Analytical solution for a circular opening in an elastic-brittle-plastic rock," International Journal of Rock Mechanics and Mining Sciences, vol. 43, no. 4, pp. 616-622, 2006.

[11] Y. Wang, "Ground response of circular tunnel in poorly consolidated rock," Journal of Geotechnical Engineering, vol. 122, no. 9, pp. 703-708, 1996.

[12] S. Q. Xu and M. H. Yu, "The effect of the intermediate principal stress on the ground response of circular openings in rock mass," Rock Mechanics \& Rock Engineering, vol. 39, no. 2, pp. 169-181, 2006.

[13] X. Chen, C. P. Tan, and C. M. Haberfield, "Numerical evaluation of the deformation behaviour of thick-walled hollow cylinders of shale," International Journal of Rock Mechanics \& Mining Sciences, vol. 37, no. 6, pp. 947-961, 2000.

[14] R. Rahimi and R. Nygaard, "Comparison of rock failure criterion in predicting borehole shear failure," International Journal of Rock Mechanics \& Mining Sciences, vol. 79, Article ID IJRMMSD1500037, 2015.

[15] W. M. Huang and X. Y. Gao, "Tresca and von mises yield criterion: a view from strain space," Philosophical Magazine Letters, vol. 84, no. 10, pp. 625-629, 2004.

[16] S. K. Sharan, "Analytical solutions for stresses and displacements around a circular opening in a generalized HoekBrown rock," International Journal of Rock Mechanics and Mining Sciences, vol. 45, no. 1, pp. 78-85, 2008.

[17] X. Chen, C. P. Tan, and C. M. Haberfield, "Solutions for the deformations and stability of elastoplastic hollow cylinders subjected to boundary pressures," International Journal for Numerical \& Analytical Methods in Geomechanics, vol. 23, no. 8, pp. 779-800, 2015.

[18] J. M. Zhu, Z. X. Wu, H. T. Zhang et al., "Study of residual stress of rock based on Lade-Duncan and SMP strength criterion," Chinese Journal of Rock Mechanics and Engineering, vol. 31, no. 8, pp. 1715-1720, 2012.

[19] X. Q. Yang, Z. Z. Zhu, H. E. Shi-Xiu et al., "Researches on failure criterion of lade-Duncan, Matsuoka-nakai and ottosen," Chinese Journal of Geotechnical Engineering, vol. 28, no. 3, pp. 337-342, 2006.
[20] P. V. Lade and M. K. Kim, "Single hardening constitutive model for frictional materials III. Comparisons with experimental data," Computers \& Geotechnics, vol. 6, no. 1, pp. 31-47, 1988.

[21] D. Ma, H. Y. Duan, X. B. Li, Z. H. Li, Z. L. Zhou, and T. B. Li, "Effects of seepage-induced erosion on nonlinear hydraulic properties of broken red sandstones," Tunnelling and Underground Space Technology, vol. 91, Article ID 102993, 2019.

[22] X. Ma, J. W. Rudnicki, and B. C. Haimson, "The application of a Matsuoka-Nakai-Lade-Duncan, failure criterion to two porous sandstones," International Journal of Rock Mechanics \& Mining Sciences, vol. 92, pp. 9-18, 2017.

[23] P. V. Lade, "Cubical triaxial tests on cohesionless soils," Soil Mechanics and Foundation Engineering, vol. 99, no. 10, pp. 793-812, 1973.

[24] A. M. Al-Ajmi and R. W. Zimmerman, "Relationship between the parameters of the Mogi and Coulomb failure criterion," International Journal of Rock Mechanics and Mining Sciences, vol. 42, no. 3, pp. 431-439, 2005.

[25] D. Ma, H. Y. Duan, Q. Zhang, J. X. Zhang, W. X. Li, Z. L. Zhou et al., "A numerical gas fracturing model of coupled thermal, flowing and mechanical effects," Computers, Materials \& Continua, vol. 65, no. 3, pp. 2123-2141, 2020.

[26] T. R. Rogge and C. F. Sieck, "He use of NONSAP to compare the Von Mises and a modified Von Mises yield criterion," Computers \& Structures, vol. 17, no. 5, pp. 705-710, 1983.

[27] H. Matsuoka and D. Sun, "Extension of spatially mobilized plane (SMP) to frictional and cohesive materials and its application to cemented sands," Soils \& Foundations, vol. 35, no. 4, pp. 63-72, 1995.

[28] D. Ma, J. J. Wang, X. Cai et al., "Effects of height/diameter ratio on failure and damage properties of granite under coupled bending and splitting deformation," Engineering Fracture Mechanics, vol. 220, Article ID 106640, 2019.

[29] D. Ma, H. Y. Duan, W. T. Liu, X. T. Ma, and M. Tao, "Watersediment two-phase flow inrush hazard in rock fractures of overburden strata during coal mining," Mine Water and the Environment, vol. 39, no. 2, pp. 308-319, 2020.

[30] C. J. Deng, G. J. He, and Y. R. Zheng, "Studies on DruckerPrager yield criterions based on $\mathrm{M}-\mathrm{C}$ yield criterion and application in geotechnical engineering," Chinese Journal of Geotechnical Engineering, vol. 28, no. 6, pp. 735-739, 2006.

[31] C. A. M. Veeken, J. V. Walters, C. J. Kenter et al., "Use of plasticity models for predicting borehole stability," International Journal of Rock Mechanics and Mining Sciences \& Geomechanics Abstracts, vol. 27, no. 6, p. 379, 1989.

[32] M. H. Yu, S. Y. Yang, S. C. Fan et al., "Unified elasto-plastic associated and non-associated constitutive model and its engineering applications," Computers \& Structures, vol. 71, no. 6, pp. 627-636, 1999.

[33] M. H. Yu, "Advances in strength theories for materials under complex stress state in the 20th century," Advances in Mechanics, vol. 34, no. 4, pp. 529-560, 2004.

[34] M. H. Yu, Unified Strength Theory and its Applications, Springer-Verlag, Berlin, Germany, 2004.

[35] R. T. Ewy, "Wellbore-stability predictions by use of a modified lade criterion," Spe Drilling \& Completion, vol. 14, no. 2, pp. 85-91, 1999.

[36] E. T. Brown, J. W. Bray, B. Ladanyi et al., "Ground response curves for rock tunnels," Journal of Geotechnical Engineering, vol. 109, no. 1, pp. 15-39, 1983.

[37] S. K. Sharan, "Elastic-brittle-plastic analysis of circular openings in Hoek-Brown media," International Journal of Rock Mechanics \& Mining Sciences, vol. 40, no. 6, pp. 817-824, 2003. 
[38] H. S. Yu, Cavity Expansion Methods in Geomechanics, Kluwer Academic Publishers, New York, NY, USA, 2000.

[39] Q. Zhang, B. S. Jiang, X. S. Wu, H. Q. Zhang, and L. J. Han, "Elasto-plastic coupling analysis of circular openings in elasto-brittle-plastic rock mass," Theoretical and Applied Fracture Mechanics, vol. 60, pp. 60-67, 2012.

[40] M. B. Reed, "Stresses and displacements around a cylindrical cavity in soft rock," Ima Journal of Applied Mathematics, vol. 36, no. 3, pp. 223-245, 1986. 